Review

\title{
Electrochemical Sensors Based on Carbon Nanotubes
}

\author{
A. J. Saleh Ahammad ${ }^{1}$, Jae-Joon Lee ${ }^{1,2, *}$ and Md. Aminur Rahman ${ }^{2, *}$ \\ 1 Department of Advanced Technology Fusion, Konkuk University, Seoul 143-701, Korea \\ 2 Department of Applied Chemistry, Konkuk University, Chungju 380-701, Korea \\ E-Mails: saleh203@konkuk.ac.kr; jjlee@kku.ac.kr; marahman@kku.ac.kr
}

* Authors to whom correspondence should be addressed; E-Mails: jjlee@kku.ac.kr(J.J.L.); marahman@kku.ac.kr (M.A.R.); Tel.: +82-43-840-3580,+82-43-840-3568; Fax: +82-43-851-4169

Received: 8 January 2009; in revised form: 17 March 2009 / Accepted: 30 March 2009 /

Published: 30 March 2009

\begin{abstract}
This review focuses on recent contributions in the development of the electrochemical sensors based on carbon nanotubes (CNTs). CNTs have unique mechanical and electronic properties, combined with chemical stability, and behave electrically as a metal or semiconductor, depending on their structure. For sensing applications, CNTs have many advantages such as small size with larger surface area, excellent electron transfer promoting ability when used as electrodes modifier in electrochemical reactions, and easy protein immobilization with retention of its activity for potential biosensors. CNTs play an important role in the performance of electrochemical biosensors, immunosensors, and DNA biosensors. Various methods have been developed for the design of sensors using CNTs in recent years. Herein we summarize the applications of CNTs in the construction of electrochemical sensors and biosensors along with other nanomaterials and conducting polymers.
\end{abstract}

Keywords: Carbon Nanotubes; Modified Electrodes; Electrochemical Sensors; Biosensors; Immunosensors; DNA sensors.

\section{Introduction}

A sensor is a device which detects a variable quantity, usually electronically, and converts the measurement into signals to be recorded elsewhere. The most important aspect of investigation of 
sensors is sensitivity, selectivity, and stability. Sensors can be classified, according to the type of energy transfer, as thermal, electromagnetic, mechanical, and electrochemical. Among them, the electrochemical sensors are very promising analytical methods because of their high degree of selectivity and sensitivity. They are more useful and easy to determine the concentrations of various analytes in samples such as fluids and dissolved solid materials. They are frequently used in clinical diagnostics, occupational safety, medical engineering, process measuring engineering, and environmental analysis.

Currently, much attention has been focused on developing nanomaterials, which are used for signal amplification in electrochemical sensors. Nanomaterials are usually used to take advantage of a larger surface area for biomolecules to be immobilized. This generally increases the number of binding sites available for the detection of a specific chemical analyte [1]. Various types of nanomaterials are used in electrochemical sensors. Carbon nanotubes (CNTs) [2] are one of the most exciting materials because of their unique electronic, chemical, and mechanical properties [3]. CNTs possessed $\mathrm{sp}^{2}$ carbon units with several nanometers in diameter and many microns in length. Two groups of CNTs, multi-walled (MW) and single-walled (SW), can be synthesized by electrical arc discharge, laser vaporization, and chemical vapor deposition methods. CNTs behave as either metals or semiconductors, depending on the diameter and the degree of helicity [4]. They are suitable for the modification of various electrodes due to their high electronic conductivity for the electron transfer reactions and better electrochemical and chemical stabilities in both aqueous and non-aqueous solutions [5]. Furthermore, construction of efficient electrochemical sensors using the CNTs-modified electrodes is very promising in that they promote electron-transfer reactions in several small biologically important molecules and large biomolecules [6,7].

This review focuses on the use of CNTs for electrochemical sensors and biosensors. Thousands of paper have been published in this field during last decade and therefore the references that appeared before 2006 were not included in this manuscript. Electrochemical detection based on a voltammetric and chronopotentiometric techniques was mostly discussed, whereas some other detection techniques related with electrophoresis, chromatography, and lab-on-a-chip were not included.

\section{Electrochemistry of Carbon Nanotubes}

CNTs are electrochemically inert materials similar to other carbon-based materials used in electrochemistry, i.e. glassy carbon, graphite, and diamond. They possess distinct electrochemical properties because of their unique electronic structure. The carbon atoms of CNTs at the sidewall and the end of the tubes are not same and their behavior can be compared with the basal plane and edge plane of highly oriented pyrolytic graphite (HOPG), respectively [8]. Compton et al. studied the redox reaction of ferricyanide at the $\mathrm{C}_{60^{-}}$and nanotube modified electrodes and compared these results with basal and edge planes pyrolytic graphite electrodes. They observed similar electron transfer rate constants for CNTs-modified and the edge plane HOPG electrodes. They reported that the CNTs acted as an efficient electron transfer promoter [Figure 1] [9-11]. To illustrate the electron transfer kinetics, Holloway et al. studied the voltammetry of two standard redox processes of $\mathrm{Fe}(\mathrm{CN})_{6}{ }^{4-}$ and $\mathrm{Ru}\left(\mathrm{NH}_{3}\right)_{6}{ }^{3+}$ using three types of MWCNTs having oxygenated edge-plane, edge-plane, and almost no edge-plane like defects [12]. The rate of electron transfer was determined to be faster in the case of edge-plane 
defect sites. This further indicates that the electroactive sites on MWCNTs are located at the tube ends. The electrochemical properties of ferricyanide redox couple at aligned and randomly dispersed SWNTs-modified electrodes was studied by Gooding et al. [13,14]. For an acid-treated aligned SWNTs-modified electrode, a peak separation of $59 \mathrm{mV}$ with a half-wave potential $\left(\mathrm{E}_{1 / 2}\right)$ of $0.231 \mathrm{~V}$ (vs. $\mathrm{Ag} / \mathrm{AgCl}$ ) for the ferricyanide redox couple was observed. On the other hand, the peak separation was observed to be $99 \mathrm{mV}$ when the acid-treated but randomly dispersed SWNTs modified electrode was used. The aligned SWNTs modified electrode shows the better electrochemical properties. It means that the electrochemistry of CNTs is dominated by the ends of the tubes.

Figure 1. Cyclic voltammograms for the reduction of $1 \mathrm{mM}$ ferricyanide for different electrodes at a scan rate of $100 \mathrm{mV} \mathrm{s}^{-1}$ [10]. Reproduced by permission of The Royal Society of Chemistry.

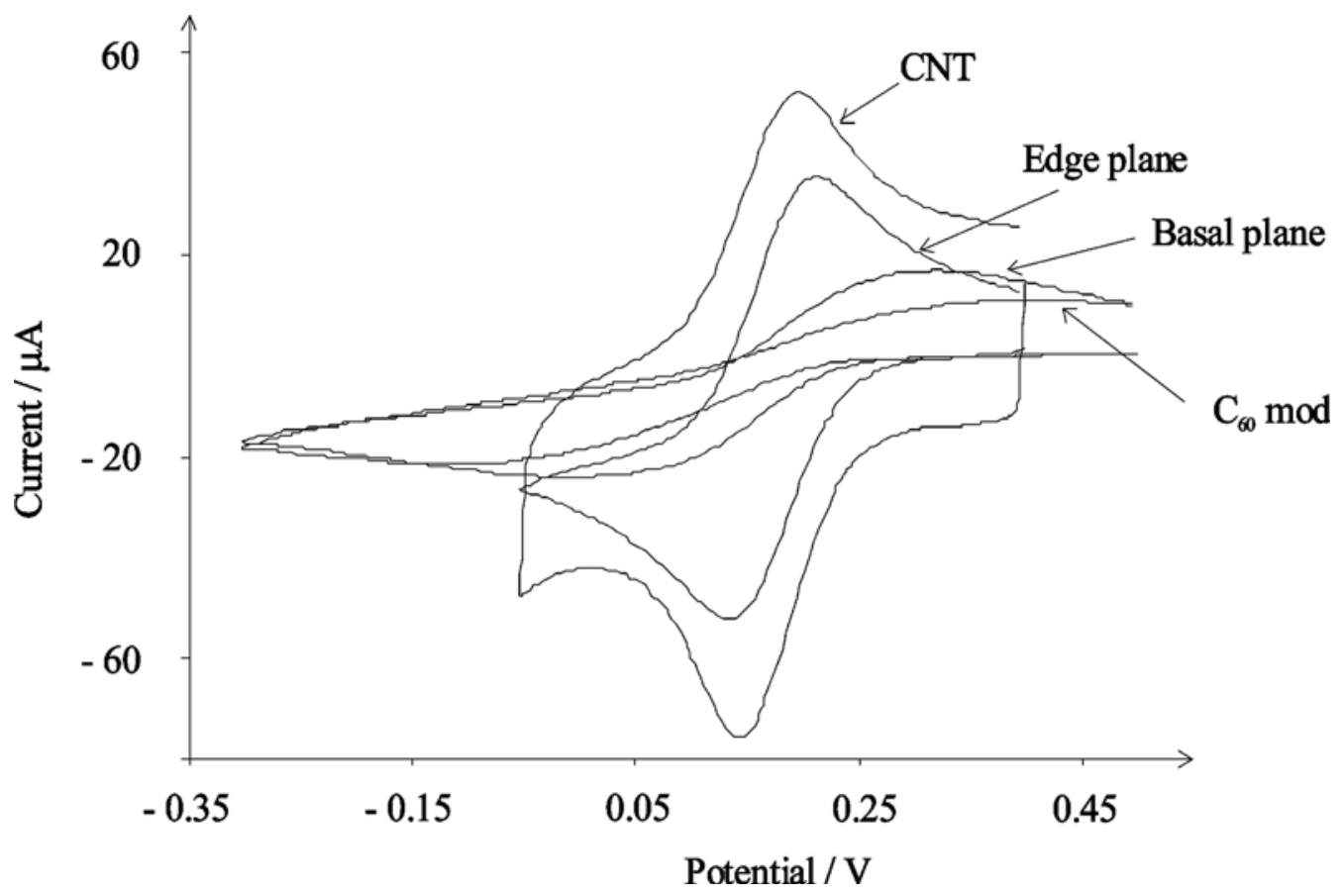

The length of the aligned CNTs also has a significant effect on the electron transfer rate. The electron transfer rate constant varied inversely with the mean length of the CNTs. Gooding et al. investigated the effect of the length of CNTs on the apparent electron transfer rate constant of the surface attached ferrocenemethylamine on to the vertically aligned CNTs with cutting times of 2, 4, and $6 \mathrm{hrs}$ during acid treatment [15]. The apparent rate constants were determined to be $98 \pm 25,187 \pm$ 98 , and $459 \pm 132 \mathrm{~s}^{-1}$ for the mean lengths of 1175,507 , and $257 \mathrm{~nm}$, respectively. When the nanotube dispersed randomly, the rate constant was found to be $12 \pm 8 \mathrm{~s}^{-1}$ for a mean tube length of $257 \mathrm{~nm}$, which was 40 times slower than that obtained at vertically aligned nanotubes modified electrode. The electron transfer rate at the CNTs modified electrode could be further increased by dialyzing the CNTs after purification and shortening treatment [16]. During purification and shortening of the SWNTs in concentrated nitric and sulfuric acids mixture, some residual acid moieties adsorbed on single-walled carbon nanotubes (SWNTs). Using TEM and HR-TEM techniques, Pumera et al. confirmed that CNTs contain residual metal impurities after acid wash with concentrated nitric acid at temperature of 
$80{ }^{\circ} \mathrm{C}$ [17]. These acid moieties can be reduced by dialyzing the purified and dialyzed CNTs against a solution of $\operatorname{Triton}^{\circledR} \mathrm{X}-100$. The electrochemical measurements using self-assembled ferrocenefunctionalized nanotube monolayers on a gold electrode showed that the dialyzed nanotubes exhibited a faster rate of electron transfer compared to the nondialyzed nanotubes [16].

The adsorbed acid moieties during purification and acid-treatment processes can also decrease the electrocatalytic activity of CNTs in electroanalysis. Pumera and his colleague suggested the use of dc magnetic susceptibility and electron paramagnetic resonance for screening and quality control of CNTs before use them in electroanalysis [18]. Recently, Dai and co-workers reported that vertically aligned nitrogen-doped CNTs can act as a metal-free electrode with a much better electrocatalytic activity [19]. The electrocatalytic activity and the electroanalytical performance at CNTs modified electrodes are strongly depended on the mode of production of the CNTs, either by chemical vapor deposition (CVD) or the ARC discharge process [20]. CNTs produced by CVD appear to be more electrochemically reactive in their voltammetric study than those produced using the ARC methodology. The differences in the electrochemical reactivity are attributed to the smaller fraction of exposed edge planes at ARC-CNTs and higher density of edge plane defects at CVD-CNTs. The electrocatalytic activity of ARC-CNTs can be increased after pre-anodization. Wang's group illustrated the effect of the electrochemical pretreatment of ARC- and CVD-prepared multi-walled CNTs using nicotinamide adenine dinucleotide, ascorbic acid, hydrazine, and hydrogen peroxide model redox systems [21]. The fact that the ARC-CNTs display a marked improvement in their electrochemical reactivity, which indicates that the pre-anodization effectively breaks the end caps of ARC-CNTs to expose new edge plane-like sites.

\section{Carbon Nanotube-Based Electrochemical Sensors}

There have been many reports on CNTs-based electrochemical sensors. Various electrochemical techniques were used for sensing of biomolecules. Some common techniques are voltammetry, amperometry, potentiometry, impedemetry, and conductometry, which have been described in our previous review paper [22]. Voltammetry, measuring the current as a response to the applied potential, is one of the most useful and widespread technique among them. For enhanced current response, it is very important to develop a stable and highly target specific interface by various surface modification of conventional electrodes. The sensitivity and the selectivity are the crucial issues for the development of sensors for detecting biologically important molecules. Much effort has been made in the development of a highly sensitive and selective method for the detection of dopamine (DA), which is one of the important catecholamine neurotransmitters in the mammalian central nervous system. Conventional electrodes are not suitable for the determination of DA due to the interference from ascorbic acid (AA) and uric acid (UA), which are co-existed in a real sample at 100 times higher concentration than DA. These compounds can be easily oxidized at the similar potential of DA and thus always interfere with DA detection. The CNTs-modified electrodes have been widely used to resolve this problem. Wang et al. reported the fabrication of a poly (3-methylthiophene) modified glassy carbon electrode (GCE) coated with Nafion/SWCNTs for highly selective and sensitive determination of DA [23]. The modified electrode enhanced the voltammetric signal of DA and effectively suppressed the interferences of AA and UA as well. A lower detection limit of $5.0 \mathrm{nM}$ was 
achieved for DA. It was also successfully applied for the determination of DA in healthy human blood serum. A CNT-polymer composite-modified electrode, with poly(styrenesulfonic acid) sodium salt and SWCNTs, were used for selective detection of DA [24]. The negatively charged poly (styrene sulfonic acid) sodium salt attracted positively charged DA in $\mathrm{pH} 7 \mathrm{PBS}$ and selectively detected it from the interference of AA.

Polymer-MWCNTs composite can be used for the fabrication of DA sensor. Yin et al. developed a DA sensor using $\beta$-cyclodextrin-incorporated MWCNTs on a polyaniline (PANI) modified GCE [25]. A superior transducing property of PANI, a rapid electron transfer capability of MWCNTs, and the preconcentration by $\beta$-cyclodextrin showed the excellent sensitivity, selectivity, stability, and reproducibility in the determination of DA. Recently, Angeles et al. also developed an amperometric sensor for DA detection by using $\beta$-cyclodextrin and MWCNTs without polymer [26]. The enhancement of the oxidation current of DA was possible due to its diffusion through the $\beta$-cyclodextrin cavities and the facile contact with the dispersed MWCNTs. Moreover, Li et al. found that polypyrrole-SWCNTs composite film can detect DA, AA, and UA simultaneously, and it showed the electrocatalytic activity towards the oxidation of nitrite [27]. Zhou's group reported that the modified electrode with poly (acrylic acid) and MWCNTs can suppress the oxidation peak of AA but enhance the DA and UA signals [28]. The application of poly (neutral red) functionalized MWCNTs was also reported by Yogeswaran and Chen for the simultaneous detection of AA, UA, and DA [29]. The clear separation of peaks was attributed to the electrostatic and hydrophobic interaction between the three analytes and the fixed cationic sites on polymer backbone as well as the functionalized MWCNTs, which are negatively charged.

Another important catecholamine neurotransmitter is epinephrine (EP), which is involved in the message transfer of the mammalian central nervous system. In biological fluids such as blood and urine, EP coexists with AA, and UA, so AA and UA may interfere during the electrochemical detection of EP at an unmodified electrode. CNTs-modified electrodes have been successfully used for the determination of EP. Chen's group developed a method for simultaneous determination of AA, EP, and UA at physiologically relevant conditions by using the composite film composed of functionalized-MWCNTs and Nafion incorporating platinum and gold nanoparticles [30]. The oxidation peaks for AA, EP, and UA were separately observed and thus, the detections of these compounds did not interfere with each other. An EP sensor prepared by an in-situ electropolymerization of brilliant cresol blue (BCB) was reported by Yi et al. [31]. In this work, the GCE modified by the film of polymeric BCB and functionalized MWCNTs composite were used to detect EP. A low detection limit of $10 \mathrm{nM}$ was obtained by using the $\mathrm{BCB}$ and functionalized MWCNTs nanocomposite. However, the authors did not discuss the issue of interference from other biological compounds such as AA. Valentini et al. used functionalized SWCNTs instead of MWCNTs for the selective detection of EP in the presence of AA [32]. They used the stainless steel microelectrodes modified by hydroxyl group functionalized SWCNTs, which were deposited electrophoretically. The presence of electron-donating-OH groups on SWCNTs repels AA and attracts the positively charged EP. It provided a relatively high electrochemical sensitivity for EP up to the detection limit of $2.0 \mathrm{nM}$.

Nicotinamide adenine dinucleotide (NADH) is a coenzyme involved in a wide range of enzymatic reactions. The direct oxidation of NADH at a bare electrode needs a high overpotential. CNT-modified 
electrode can be used for the stable low potential detection of NADH. Zhai et al. developed a multilayer film of MWCNTs and chitosan (CS) using the layer-by-layer (LBL) method by taking advantage of the interaction between a positively charged CS and the negatively charged MWCNTs [33]. They assembled nine-layers of CS/MWCNTs successfully, which showed a very rapid and stable response of $\mathrm{NADH}$ oxidation at about $400 \mathrm{mV}$ with the detection limit $(\mathrm{S} / \mathrm{N}=3)$ of $0.3 \mu \mathrm{M}$. A layerby-layer approach is an efficient way to increase the amount of catalyst or enzyme at the sensor surface. However, the thickness of the multilayer need to be optimized as the sensor response can be suppressed by the very thick multilayer. Wang et al. fabricated an electrode by mixing a roomtemperature ionic liquid, 1-butyl-3-methylimidazolium tetrafluoroborate, and MWCNTs along with CS. This electrode was used for NADH sensing with the detection limit of ca. $0.06 \mu \mathrm{M}$ [34]. The use of ionic liquid and MWCNTs at the sensor surface may increase the surface ionic and electrical conductivities, thus, may enhance the sensitivity of the sensor. Radoi et al. used the covalently linked variamine blue, as a redox mediator to the oxidized SWCNTs for the detection of NADH [35]. The NADH oxidation potential was found to be lowered, from the changes of formal redox potential of the mediator, and therefore the sensor efficiency was improved due to the electrocatalytic activity of the mediator. Another very common approach for NADH sensors is the use of polymer composites with MWCNTs such as poly (acrylic acid)-wrapped MWCNTs complex [36], MWCNTs-poly (1,2diaminobenzene) nanoporous composite [37], quinone-amine polymer- MWCNTs nanocomposite [38], and poly-(3-methylthiophene)-MWCNTs hybrid composite [39]. The sensitivities of the polymerMWCNTs nanocomposite-based NADH detections were found to be enhanced due to the excellent electrocatalytic activities of the nanocomposites.

Hydrogen peroxide $\left(\mathrm{H}_{2} \mathrm{O}_{2}\right)$ is a product of several biological, enzyme-catalyzed reactions. The detection of $\mathrm{H}_{2} \mathrm{O}_{2}$ plays an important role in food industry, environmental protection, and in medical diagnostics. For the sensitive detection of $\mathrm{H}_{2} \mathrm{O}_{2}$, Tkac and Ruzgas have used an electrode modified with SWCNTs. The sensitivity was highly dependent on the dispersing agent in the organic solvents and charging status of polymers (e.g. Nafion and CS) [40]. They found that the dispersion of both polymers is highly stable but the SWCNTs in the CS dispersion showed higher sensitivity for $\mathrm{H}_{2} \mathrm{O}_{2}$ compared to that in Nafion. Sun et al. introduced the modification with the ferrocene-filled SWCNTs for a $\mathrm{H}_{2} \mathrm{O}_{2}$ sensor with a good stability and reproducibility [41]. Ferrocene/ferrocenium $\left(\mathrm{Fc} / \mathrm{Fc}^{+}\right)$was used as an electron-transfer mediator for the redox reaction of $\mathrm{H}_{2} \mathrm{O}_{2}$.

Electrochemical detections of metal ions have widely been studied using CNTs-modified electrode. For example, Yuan et al. developed a mercury-free electrode system by casting a dispersed solution of MWCNTs in Nafion on GCE [42]. It was applied for the detection of europium (III) by differential pulse adsorptive stripping voltammetry (DPASV) and a wide linear range from $40 \mathrm{nM}$ to $10 \mathrm{mM}$ with lower detection limit of $10 \mathrm{nM}$ was obtained. Sun et al. used SWCNTs-Nafion film for the determination of $\mathrm{Cd}^{2+}$ in water [43]. Profumo et al. have prepared chemically modified MWCNTs electrode to detect the trace amount of As (III) and Bi (III) in a natural and high-salinity waters [44]. An oxidation of MWCNTs to introduce carboxyl groups and the subsequent chemical treatments were required to get a robust modification of the electrode surface for reliable measurement of saline water without desalting the sample (Figure 2). 
Figure 2. Scheme for the preparation of MWCNTs chemically modified electrode bearing SH groups. Reprinted with permission from [44]. Copyright (2006) American Chemical Society.

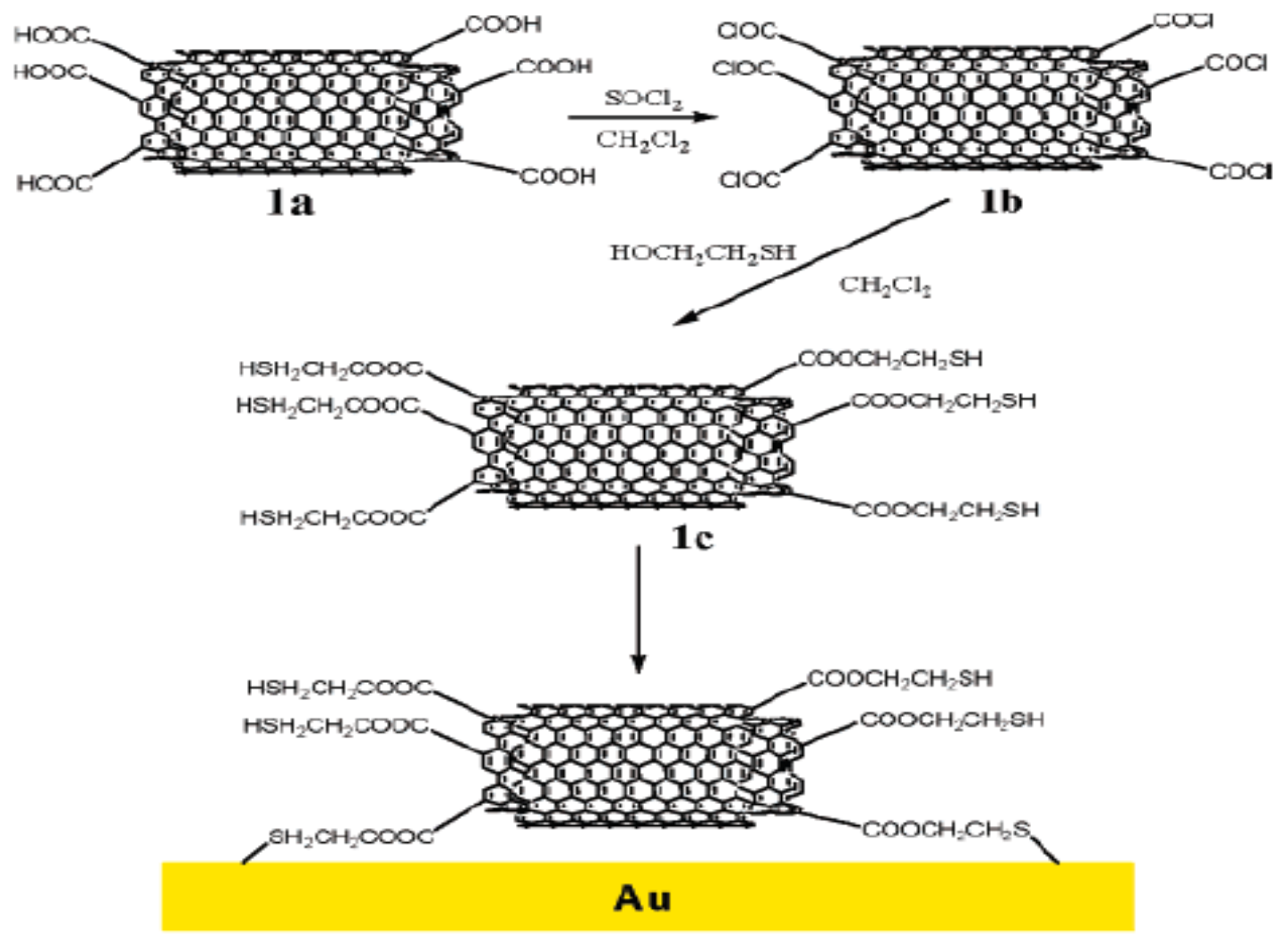

Phosphate containing molecules such as phytic acid (PA), phosphomolybdic acid (PMA), dihexadecylphosphate (DHP), and dicetyl phosphate (DCP) have been widely used with CNTs for sensing applications. Jeon's group recently developed an electrochemical sensor based on the modification of platinum electrode with SWCNTs and PA for the selective detection of DA in the presence of AA and UA [45]. The PA-SWCNTs films promoted the electron transfer reaction of DA while the PA in the films act as a binder and a negatively charged linker as well. The PA-SWCNTs/Pt electrode can separate the oxidation peak of DA from the interferences of electrochemical responses of AA and UA. Li et al. used PMA with MWCNTs to make an amperometric bromate sensor [46]. Hu and co-workers described a method for the preparation of a MWCNTs-DHP composite film on the GCE surface for the determination of lincomycin in tablets [47]. They found a well-defined oxidation peak for lincomycin by using cyclic voltammetry $(\mathrm{CV})$ and a linear response range from $0.45 \mu \mathrm{M}$ to $0.15 \mathrm{mM}$ with the detection limit of $0.2 \mu \mathrm{M}$. The similar approach was applied by Ming et al. recently for the determination of trace Sudan I contamination in chili powder [48] and the same electrode was also used to determine the acyclovir voltammetrically [49]. DHP was replaced by DCP and 4aminobenzenesulfonic acid (4-ABSA) for the determination of 2-chlorophenol [50] and tyrosine [51], respectively. The GCE modified with MWCNTs even without DHP was also used for the determination of procaine [52] and phenylephrine [53]. 
Rutin is a kind of flavonoid glycoside that has a wide range of physiological activities such as antiinflammatory, antitumor, and antibacterial. CNT modified electrodes have been successfully using for the determination of rutin. A gold electrode modified with SWCNTs was fabricated, by Zeng et al., to investigate the voltammetric behavior of rutin [54]. At this electrode rutin exhibited an adsorptioncontrolled electrode reaction with a pair of peaks at $\sim 0.35 \mathrm{~V}$, implying two electron transfer process at the electrode surface. The linear range from $20 \mathrm{nM}$ to $5.0 \mu \mathrm{M}$ and the detection limit of $10 \mathrm{nM}$ were determined. Yu's group developed a rutin sensor based on GCE modified with $\beta$-cyclodextrin incorporated MWCNTs to take advantage of the inclusion interaction of $\beta$-cyclodextrin and rutin [55]. GCE modified by SWCNTs/poly (Neutral Red) composite film also exhibited a good catalytic activity on the redox properties of rutin [56]. Rutin, a kind of mediator was also used with MWCNTs for the fabrication of a hydroxylamine sensor [57].

Metal nanoparticles have received considerable attention in recent years. Incorporation of nanoparticles to CNTs for the modification of electrodes has been demonstrated to enhance the electrocatalytic activity of many electrochemical processes and therefore be suitable for sensing applications. Hrapovic et al. focused on metal nanoparticles/CNTs nanocomposites for electrochemical detection of trinitrotoluene (TNT) and other nitroaromatics [58]. They found that $\mathrm{Cu}$ nanoparticles and SWCNTs solubilized in Nafion provided the highest sensitivity for TNT with the detection limit of 1 ppb for analysis of TNT in tap water, river water, and contaminated soil. The composites of SWCNTs, gold nanoparticle (GNP), and ionic liquid (i.e. 1-octyl-3-methylimidazolium hexafluorophosphate) were used to fabricate a modified GCE for the sensitive voltammetric detection of chloramphenicol [59]. The composition of the film and the operation conditions played an important role in the voltammetric response and the detection limit under optimum condition was $5.0 \mathrm{nM}$. Electrochemically deposited Pt nano-clusters onto MWCNTs-modified GCE showed a strong electrocatalytic activity toward the oxidation of estrogens involving estradiol, estrone, and estriol [60]. This electrode showed the linear responses between $0.5-15 \mu \mathrm{M}, 2.0-50 \mu \mathrm{M}$, and 1.0-75 $\mu \mathrm{M}$ for estradiol, estrone, and estriol, respectively, in the square-wave voltammetry. Wei and co-workers used composite of nano-silver coated MWCNTs to determine a trace of thiocyanate in urine and saliva samples from smokers and nonsmokers and observed the decrease of anodic current upon addition of trace amount of thiocyanate in $\mathrm{nM}$ level [61]. Liu et al. designed a sensor by electropolymerization of thionine at the GCE modified with GNPs/MWCNTs composites for simultaneous determination of adenine and guanine in DNA [62]. The detection limits $(\mathrm{S} / \mathrm{N}=3)$ of $10 \mathrm{nM}$ and $8.0 \mathrm{nM}$ were obtained for guanine and adenine, respectively.

Carbon-paste electrodes (CPEs) have been widely used as a suitable matrix for preparation of modified electrodes due to their simple preparation, renewability, and compatibility with various types of modifiers. CNT-modified CPEs showed considerable improvements in electrochemical behavior of materials. Zhuang et al. reported the fabrication of CPE modified with MWCNTs for the determination of bergenin using CV and differential pulse voltammetry (DPV) [63]. They found that the oxidation peak current of bergenin increased significantly upon increase of the contents of MWCNT and the linear dynamic range of $0.6 \sim 10 \mu \mathrm{M}$ was observed at an optimum experimental condition with a detection limit of $70 \mathrm{nM}$. Shahrokhian and Fotouhi added cobalt salophen (CoSal) for the determination of tryptophan [64]. The MWCNTs/CoSal CPE exhibited an electrocatalytic activity on the oxidation of tryptophan. Furthermore, this electrode could be used to detect tryptophan separately 
from the interferences of AA and cysteine. They also showed Nafion-incorporated MWCNTs/CoSal modified CPE can be used for the simultaneous detection of UA and AA. The detection limits were determined to be 60 and $100 \mathrm{nM}$, respectively [65]. When thionine-Nafion was incorporated into MWCNTs/CPE, it could be used to detect DA and AA simultaneously [66]. Recently, an electrode coated with SWCNTs paste was developed for sensitive voltammetric detection of methylparathion [67]. Fan et al. fabricated such electrode using ionic liquid (1-butyl-3-methylimidazolium hexafluophosphate) as a binder and they found a good electro-catalytic behavior to the electrochemical reduction of methylparathion. It was also found that the same electrode can be used to detect methylparathion and its hydrolysate ( $p$-nitrophenol) simultaneously. The similar approach was applied for the voltammetric determination of xanthine [68] and UA [69]. A highly sensitive and fast responding electrochemical sensor was also prepared for piroxicam with MWCNTs paste electrodes by Abbaspour and Mirzajani [70]. It exhibited a high catalytic activity towards electrooxidation of piroxicam in a mild $\mathrm{pH}$ medium, showing the linear response range of $0.15-5 \mu \mathrm{g} / \mathrm{mL}$, with the detection limit of $0.1 \mu \mathrm{g} / \mathrm{mL}$. The same electrode was applied for the determinations of urapidil [71] and quercetin [72].

Composite film of CNTs with other materials such as conducting polymers, ceramics etc are very attractive combination of materials for the development of electrochemical sensors. Yang et al. fabricated a novel electrode using a poly(acid chrome blue K)/ MWCNTs composite film modified GCE for simultaneous detection of dihydroxybenzene isomers in real water samples by applying the first order linear sweep derivative voltammetry [73]. The detection limits for hydroquinone, catechol, and resorcinol were 100,100, and $90 \mathrm{nM}$, respectively with this electrode. Wang et al. developed a novel sensor system by electrochemical oxidation of the mixture of L-cysteine and CNTs at GCE for the determination of terbinafine in human serum [74]. A composite film, found on GCE after oxidation, was responsible for the significant increase in the current response of terbinafine. It showed a wide range of linear response, $80 \mathrm{nM}-50 \mu \mathrm{M}$, with the detection limit of $25 \mathrm{nM}$ for terbinafine determination. Zhu et al. prepared CNTs ceramic composites electrode for electrochemical sensing by dispersing MWCNTs into the methyltrimethoxysilane derived sol-gel solution [75]. The electrode provided a better reversible behavior with a substantial decrease of overpotential, and a higher sensitivity compared to the unmodified one towards the electrochemical detection of ascorbic acid, uric acid, acetaminophenol, NADH, EP, DA, cysteine, and $\mathrm{H}_{2} \mathrm{O}_{2}$. Wang and coworkers have prepared a RuOx/CNTs-modified GCE by drop and dry process [76]. This electrode showed an electrocatalytic activity towards insulin and exhibited a wide range of linear response of $10-800 \mathrm{nM}$ with a detection limit of $1 \mathrm{nM}$. Recently, Snider et al. developed a MWCNTs/dihydropyran composite film for the electrochemical detection of insulin secreted by islets in a microfluidic system [77]. Vega et al. developed a tetracycline antibiotic sensor based on MWCNTs modification of GCE [78]. A highly sensitive and reproducible electroanalytical response of tetracycline was attributed to the antifouling capability of the MWCNTs. Rezaei and Zare described a simple and highly sensitive method based on the modification of GCE by MWCNTs for the direct voltammetric determination of noscapine in pharmaceutical and clinical samples [79]. It significantly enhanced the noscapine signal and showed a wide linear range of $0.4 \mu \mathrm{M}-10 \mathrm{mM}$, under the optimum condition, with the detection limit of $80 \mathrm{nM}$. They also applied the same electrode to study the determination of captopril with effective electrocatalysts, hexacyanoferrate (II) [80]. The system of MWCNTs and hexacyanoferrate strongly 
enhances the oxidation of captopril and a detection limit of $0.2 \mu \mathrm{M}$ was obtained. Buratti et al. introduced cobalt oxide with the MWCNTs modified GCE for the detection of carbohydrates and thiols [81]. The modified electrode showed excellent electrocatalytic activity towards the oxidation of carbohydrates and thiols.

\section{Carbon Nanotube-Based Electrochemical Biosensors}

An electrochemical biosensor is an analytical tool for sensitive and selective detection of biomolecules. Increasing attention has been given to biosensors due to their potential applications in clinical chemistry, food industry, and environmental fields. Glucose oxidase (GOx)-based glucose biosensors are still considered as a primary model system in the development of new sensing materials and methods. They are the most extensively studied enzyme biosensors because of their high demand for blood glucose monitoring. A summary of recent progress in the field of glucose biosensors can be found in two excellent recent reviews by Wang and Heller $[82,83]$. CNTs are extremely attractive for fabricating electrochemical biosensors due to their outstanding properties, especially the excellent conductive, adsorptive and biocompatibility. Vertically aligned CNTs can be coupled with enzymes to provide a favorable surface orientation and act as an electrical connector between their redox center and the electrode surface [82]. Figure 3 showed the assembly of the CNT electrically contacted GOx electrode. Plugging enzymes into the CNTs by this way is an extremely efficient approach for the development of glucose biosensor.

Different types of glucose biosensors have been developed in recent years. Tsai and co-workers developed a nanobiocomposite film by incorporating functionalized MWCNTs and GOx into polypyrrole (PPy) film for a highly sensitive glucose biosensor [84]. The amperometric response of the optimized biosensor displayed a sensitivity of $95 \mathrm{nA} / \mathrm{mM}$, a linear range up to $4 \mathrm{mM}$, and a response time of about $8 \mathrm{sec}$. Huang et al. loaded MWCNTs and GOx on a graphite disk using a LBL assembly technique to construct a glucose biosensor [85]. The current response to glucose was highly dependent on the number of layers and the maximum response was obtained at 6 layers of MWCNTs/GOx with the detection limit of $90 \mu \mathrm{M}$. Liu and Lin also applied LBL assembly technique to construct a sandwich-like structure, PDDA/GOx/PDDA/CNTs, for a reproducible and stable glucose biosensor while Zhao and $\mathrm{Ju}$ added poly (sodium 4-styrenesulfonate) with PDDA to construct multilayer membranes [86,87]. They modified gold electrode with 3-mercapto-1-propanesulfonic-acid and then bilayers of the PDDA and PSS were formed on the modified Au surface. PDDA wrapped MWCNTs and GOx was then assembled through LBL technique. Wang et al. functionalized gold electrodes with the negatively charged 11-mercaptoundecanoic acid (MUA) and then apply the LBL assembly of a positively charged redox polymer, poly [(vinylpyridine)Os(bipyridyl $\left.)_{2} \mathrm{Cl}^{2+/ 3+}\right]$, and the negatively charged GOx/SWCNTs for glucose sensor. Liu et al. developed an amperometric glucose biosensor based on electrostatic assembly of GNPs/MWCNTs/GOx [88]. Positively charged poly (dimethyldiallylammonium chloride) was used to connect them in a LBL pattern. The electrode showed an excellent electrocatalytic activity for glucose sensing at a relatively low potential $(-0.2 \mathrm{~V})$. 
Figure 3. Assembly of the CNT electrically contacted glucose oxidase electrode. Reprinted with permission from [82]. Copyright (2008) American Chemical Society.

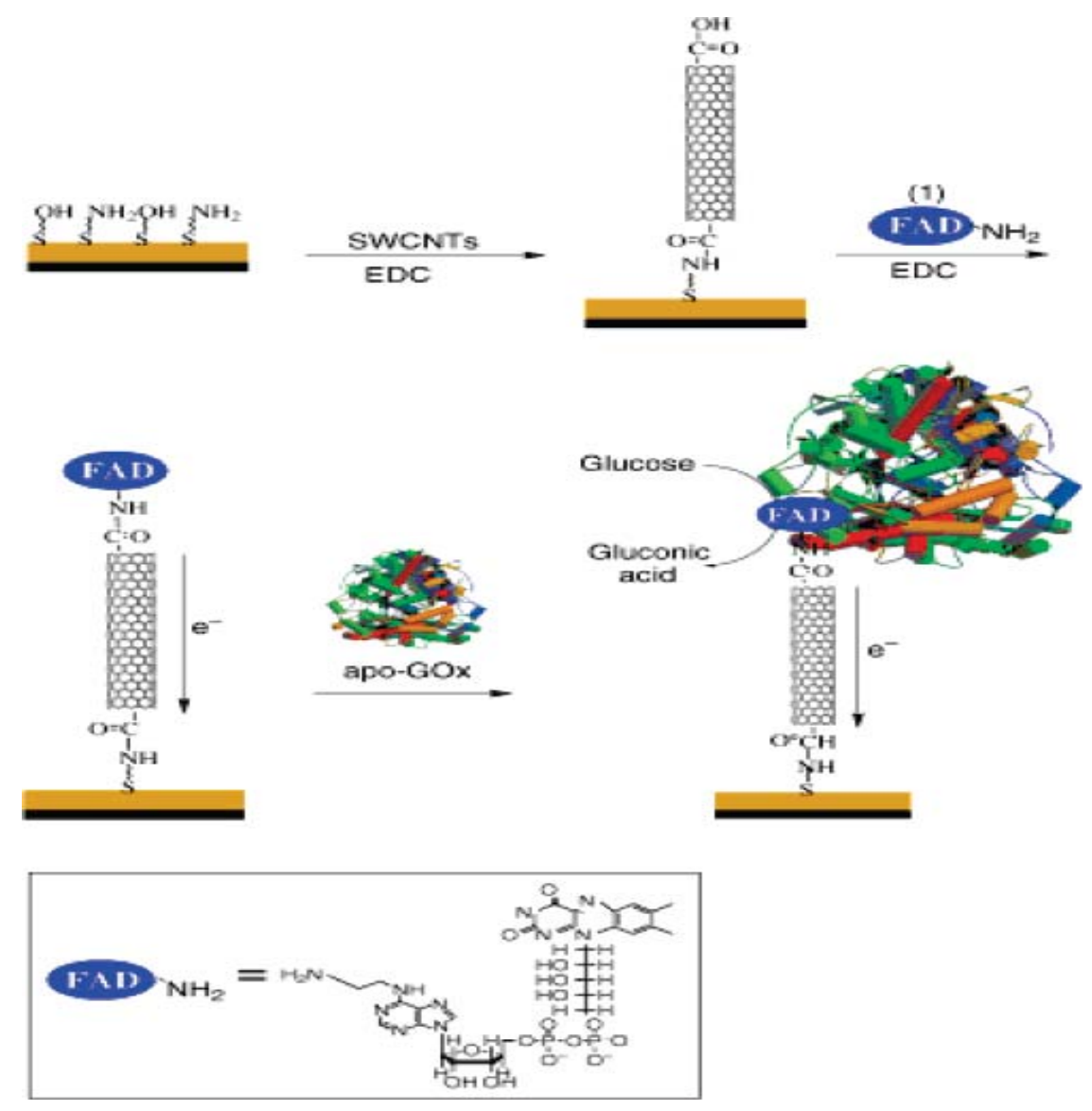

$\mathrm{Xu}$ et al. described an amperometric glucose biosensor based on an alternating electrostatic selfassembling GOx and dendrimer-encapsulated Pt nanoparticles (Pt-DENs) on MWCNTs [89]. The excellent electrocatalytic activity of CNTs and Pt-DENs toward $\mathrm{H}_{2} \mathrm{O}_{2}$ and special three-dimensional structure of the enzyme electrode resulted in a low detection limit with a wide linear response range, a high sensitivity with a good precision, and an enhanced operational stability. Shirsat et al. fabricated an amperometric glucose biosensor by applying a LBL assembly of SWCNTs and PPy multilayer film on a platinum coated with polyvinylidene fluoride (PVDF) membrane [90]. GOx was immobilized on the film by cross linking through glutaraldehyde (GA) (0.1\%) and a linear response range from $1 \mathrm{mM}$ to $50 \mathrm{mM}$ of glucose concentration with the sensitivity of $7.06 \mathrm{uA} / \mathrm{mM}$ was achieved. A glucose sensor based on the LBL assembly of functionalized MWCNTs and poly (neutral red, PNR) multilayer film was also suggested [91]. This electrode showed a significant improvement of redox activity showing a synergic effect of excellent electron-transfer capability of CNTs and PNR. Another type of glucose biosensor was constructed by immobilizing GOx onto the electrode surface using GA. Yao and Shiu constructed a mediator type glucose sensor based on the immobilization of GOx at electropolymerized poly (toluidine blue O) film on CNTs modified GCE [92]. Poly (toluidine blue O) provided the polymer matrices to maintain the sensing activity and served as a redox mediator for enzymatic glucose oxidation. This biosensor showed enhanced current response at low potential $(-0.1 \mathrm{~V})$ and therefore common interferences from AA, UA, and acetaminophen could be avoided. Zhu and coworkers suggested a Prussian Blue (PB) based amperometric glucose biosensor by assembling the PB 
nanoparticles on the surface of MWCNTs modified GCE followed by immobilization of GOx [93]. It showed good sensitivity, fast response with a detection limit of $12.7 \mathrm{mM}$. Similar PB based glucose biosensors were also prepared by immobilizing GOx in a film of LBL assembly of CS and MWCNTs [94] and on the nanocomposite film of PB nanoparticles/MWCNTs/poly (1,2-diaminobenzene) [95]. Manesh et al. fabricated a glucose biosensor based on the immobilization of GOx into an electrospun composite membrane consisting of polymethylmethacrylate (PMMA) dispersed with MWCNTs wrapped by a cationic PDDA polymer [96]. This nanofibrous electrode exhibited excellent electrocatalytic activity towards $\mathrm{H}_{2} \mathrm{O}_{2}$ with a pronounced oxidation current at $+100 \mathrm{mV}$. Glucose was detected amperometrically with this nanofibrous electrode with a detection limit of $1 \mu \mathrm{M}$. A highly sensitive and selective glucose biosensor based on immobilization of GOx within mesoporous CNTstitania-Nafion composite film coated on a platinized GCE, was also developed recently by Lee and coworkers [97]. It responded linearly to glucose in the wide range from $50 \mu \mathrm{M}$ to $5.0 \mathrm{mM}$ with sensitivity of $154 \mathrm{~mA} \mathrm{M}^{-1} \mathrm{~cm}^{-2}$.

A mediatorless glucose biosensor, based on the incorporation of GOx to the composite electrode of colloidal gold-CNTs-Teflon showed a remarkably higher sensitivity than that achieved with other GOx-CNTs bioelectrodes [98]. It could be used for ethanol biosensor by incorporating alcohol dehydrogenase (ADH) [99]. Chu et al. developed an amperometric glucose biosensor based on adsorption of GOx at the gold and platinum nanoparticles modified CNTs electrode where CNTs were covalently immobilized on cysteamine modified gold electrode [100]. The GOx/Au nano/Pt nano/CNTs/Au electrode was then covered with a thin layer of Nafion to avoid the loss of GOx and suppress the interfering signals from UA and AA. Recently, Zou et al. developed an amperometric glucose biosensor based on electrodeposition of platinum nanoparticles (PtNPs) onto MWCNTs and entrapping an enzyme in $\mathrm{CS}_{-} \mathrm{SiO}_{2}$ sol-gel [101]. This electrode showed an excellent electrocatalytic activity and high stability as well due to the synergistic action of Pt and MWCNTs and the biocompatibility of $\mathrm{CS}_{-} \mathrm{SiO}_{2}$ sol-gel. A wide linear range from $1 \mu \mathrm{M}$ to $23 \mathrm{mM}$ and a low detection limit of $1 \mu \mathrm{M}$ was achieved for glucose sensing. Zhao et al. recently investigated an amperometric glucose biosensor based on PtNPs combined aligned CNTs electrode [102]. The combination of PtNPs and CNTs in this glucose biosensor showed a highly sensitive detection of glucose. Kang et al. constructed another glucose biosensor based on the integration of CNTs with gold-platinum alloy nanoparticles (Au-PtNPs) [103]. In this sensor, GOx was immobilized in biocompatible CS through cross-linking with GA on the $\mathrm{Au}-\mathrm{PtNPs} / \mathrm{CNTs} / \mathrm{CS}$ film. It showed a low potential $(0.1 \mathrm{~V})$ detection of glucose with high sensitivity, low detection limit, good reproducibility, long-term stability, fast response, and high specificity. This biosensor was applied in the determination of glucose in real blood and urine samples with satisfactory results. Yang et al. prepared MWCNTs composite using Pt-NP doped sol/gel solution as a binder and incorporated GOx for glucose biosensor [104]. The sensitivity enhanced 4 times when Pt nanoparticles were loaded. A glucose biosensor, developed by Rivas and co-workers was based on the electrocatalytic activity of copper and iridium microparticles incorporated within the CNTs paste electrode containing GOx [105]. This biosensor detected glucose at very low potentials $(-0.1 \mathrm{~V})$ with high sensitivity and selectivity. Yao and Shiu examined the electrochemical and electrocatalytic properties of different types of CNTs material and used them for fabricating glucose biosensors [106]. They found that the electrodes modified with SWCNTs usually had better electron-transfer and electrocatalytic properties than the corresponding MWCNTs-modified 
electrodes. Recently, Jia et al. reported the fabrication of needle-type glucose biosensor by packing a mixture of MWCNTs, graphite powder, and freeze-dried GOx powder into a glass capillary of $0.5 \mathrm{~mm}$ inner diameter [107]. It showed an improved sensitivity and stability when the experimental condition was optimized. Zhu and co-workers proposed a bienzymatic mediatorless glucose biosensor based on co-immobilization of GOx and horseradish peroxidase (HRP) in an electropolymerized PPy film on a SWCNTs modified electrode [108]. They took advantage of direct electron transfer characteristics of HRP with CNTs electrode and realized a lower operational potential for selective determination with a minimized interference.

The detection of hydrogen peroxide $\left(\mathrm{H}_{2} \mathrm{O}_{2}\right)$ is very important because many enzymatic biosensors rely on the detection of $\mathrm{H}_{2} \mathrm{O}_{2}$ generated by an enzymatic reaction. Since the amount of generated $\mathrm{H}_{2} \mathrm{O}_{2}$ from an enzymatic reaction is very low, the fabrication of a highly sensitive $\mathrm{H}_{2} \mathrm{O}_{2}$ biosensor is needed. CNTs can be used in the fabrication of highly sensitive $\mathrm{H}_{2} \mathrm{O}_{2}$ biosensors. There have been many reports on CNTs based $\mathrm{H}_{2} \mathrm{O}_{2}$ biosensors. Chen and $\mathrm{Lu}$ reported the encapsulation of hemoglobin $(\mathrm{Hb})$ in the composite film of carboxylic acid functionalized MWCNTs and polyelectrolyte-surfactant polymer to develop a $\mathrm{H}_{2} \mathrm{O}_{2}$ biosensor [109]. Faradic response of the $\mathrm{Hb}$ was observed and it exhibited excellent electrocatalytic activity to reduce $\mathrm{H}_{2} \mathrm{O}_{2}$. Chen et al. proposed an amperometric thirdgeneration $\mathrm{H}_{2} \mathrm{O}_{2}$ biosensor based on the immobilization of $\mathrm{Hb}$ on the nanohybrid film of MWCNTs and gold colloidal nanoparticles [110]. A wide range of linear response from $0.21 \mu \mathrm{M}$ to $3.0 \mathrm{mM}$ with a detection limit of $80 \mathrm{nM}$ was obtained. Tripathi et al. entrapped HRP in an ormosil composite doped with ferrocene monocarboxylic acid-bovine serum albumin conjugate and MWCNTs for a $\mathrm{H}_{2} \mathrm{O}_{2}$ biosensor [111]. MWCNTs improved the conductivity of the composite film and HRP provided a fast amperometric response to $\mathrm{H}_{2} \mathrm{O}_{2}$. A wide linear range between $20 \mu \mathrm{M}$ and $4.0 \mathrm{mM}$ with a detection limit of $5.0 \mu \mathrm{M}(\mathrm{S} / \mathrm{N}=3)$ was achieved. Luo et al. developed a $\mathrm{H}_{2} \mathrm{O}_{2}$ biosensor with an improved performance based on the immobilization of HRP onto electropolymerized PANI films doped with CNTs [112]. It was found that the existence of CNTs in the biosensor system could effectively increase the amount and stability of the immobilized HRP as well as the performance of the biosensor. A $\mathrm{H}_{2} \mathrm{O}_{2}$ biosensor based on the modification of graphite electrode with toluidine blue $(\mathrm{Tb})$ modified MWCNTs was developed by H. Ju and co-workers [113]. HRP was immobilized with the aid of CS for sensing $\mathrm{H}_{2} \mathrm{O}_{2}$ with a good stability and reproducibility. Qian and Yang developed a mediator free amperometric biosensor for $\mathrm{H}_{2} \mathrm{O}_{2}$ based on composite film of MWCNTs/CS [114]. HRP was crosslinked with composite film using GA. Sanchez et al. reported the fabrication of MWCNTs/polysulfone biocomposite membrane, which allows the incorporation of HRP enzyme by phase inversion technique. This biocomposite membrane was used for the construction of a $\mathrm{H}_{2} \mathrm{O}_{2}$ biosensor [115]. Qu et al. took the combined advantages of CNTs and nano- $\mathrm{Fe}_{3} \mathrm{O}_{4}$ to prepare a magnetic $\mathrm{CNTs} /$ nano- $\mathrm{Fe}_{3} \mathrm{O}_{4}$ composite by co-precipitation. It exhibited higher electrocatalytic activity toward the redox processes of $\mathrm{H}_{2} \mathrm{O}_{2}$ [116]. They also introduced CS into the bulk of the composite by co-precipitation to immobilize GOx covalently to make an amperometric glucose sensor.

Choi et al. constructed a highly sensitive and stable amperometric ethanol biosensor based on the immobilization of $\mathrm{ADH}$ within a thin composite film of CNTs-titania-Nafion [117]. Due to the mesoporous nature of this composite film, the present ethanol biosensor exhibited remarkably fast response time and wide linear response range. Santos et al. constructed an amperometric ethanol biosensor based on co-immobilization of ADH and Methylene Blue (MB) on MWCNTs through the 
cross-linking with GA and agglutination with mineral oil [118]. The amperometric response of this biosensor showed an excellent operational stability and wide linear response range. Cai and coworkers fabricated a nanocomposite by the functionalization of SWCNTs with poly(Nile Blue A) for ethanol biosensor [119]. Immobilization of ADH onto the modified electrode showed electrocatalytic activity toward the oxidation of ethanol with a good stability, reproducibility, and higher biological affinity. Liu and Cai developed an ethanol biosensor based on the nanocomposites of positively charged PDDA wrapped SWCNTs [120]. The negatively charged ADH was immobilized on the nanocomposites via the charge interaction and this biosensor provided a good electrocatalytic activity toward the oxidation of ethanol with a good stability, reproducibility, and high biological affinity.

Yang et al. reported the modification of gold electrodes by self-assembling the positively charged Pt nanoparticle-MWCNTs-CS and negatively charged poly (sodium- $p$-styrenesulfonate) salt (PSS) for a sensitive cholesterol biosensor [121]. MWCNTs were dispersed in the Pt nanoparticle-doped CS solution to obtained Pt-CNTs-CHIT material. Cholesterol oxidase was immobilized onto the modified electrode surface using GA.

Tang et al. developed an amperometric glutamate biosensor based on self-assembly of glutamate dehydrogenase (GLDH) and poly (amidoamine) dendrimer-encapsulated platinum nanoparticles (PtPAMAM) onto carboxylic acid group-functionalized MWCNTs [122]. The modified electrode showed electrocatalytic activity toward the oxidation of glutamate with a good reproducibility and high sensitivity. Lin's group fabricated a simple and inexpensive choline biosensor based on the immobilization of choline oxidase (ChO), $\mathrm{ChO}$ and HRP bienzymes onto MWCNTs modified GCE using LBL assembly technique [123]. With this configuration, a wide linear response range from 50 $\mu \mathrm{M}$ to $5.0 \mathrm{mM}$ with a detection limit of about $10 \mu \mathrm{M}$ for choline was achieved. Song et al. fabricated an another choline biosensor by immobilizing ChO into a sol-gel silicate film on MWCNTs modified platinum electrode [124]. This biosensor was used to detect choline released from lecithin by phospholipase D (PLD) in serum samples with high sensitivity and the detection limit was $0.1 \mu \mathrm{M}$. A stable and sensitive acetylthiocholine sensor based on immobilization of acetylcholinesterase (AChE) on the CS-MWCNTs composite was developed by Du et al. [125]. GA was used as cross linker to covalently bond $\mathrm{AChE}$ and efficiently prevent leakage of the enzyme from the film.

Lee et al. developed an amperometric tyrosinase biosensor based on MWCNTs dispersed in mesoporous composite films of sol-gel-derived titania and Nafion [126]. Tyrosinase was immobilized within the composite film and phenolic compounds were determined by the direct reduction of biocatalytically liberated quinone species. This sensor exhibited remarkably fast response time less than $3 \mathrm{sec}$ and a good performance in terms of the sensitivity $(417 \mathrm{~mA} / \mathrm{M})$ and the detection limit $(0.95$ $\mathrm{nM}$ ) due to the large pore size of the composite film. An amperometric biosensor was based on the immobilization of HRP on MB modified MWCNTs for phenolic compounds and it showed a very wide linear response with a good sensitivity for catechol [127]. Recently, Lopez et al. described a biosensor fabricated by the modification of GCE with a matrix based on MWCNTs, tetrahydrofuran (THF) mixed with poly(vinylchloride) (PVC), and with a GA solution (MWCNTs-TPG/GC) for NADH detection [128]. The modified electrode showed a relatively higher sensitivity, a promotion of electron transfer, and it facilitated the amperometric determination of NADH starting in a potential of $+0.40 \mathrm{~V}$. Male et al. developed a biosensor for arsenite by depositing molybdenum-containing arsenite oxidase galvanostatically onto the active surface of a MWCNTs on GCE [129]. The detection limit of 
$1 \mathrm{ppb}$ was found for arsenite but there was a severe interference caused by common metal ions found in tap and river waters. Mita et al. constructed a bisphenol A biosensor using a various tyrosinase containing CPE and they optimized the experimental condition with the composition of $10 \%$ tyrosinase, 45\% SWCNTs, and 45\% mineral oil [130]. It showed a good reproducibility with a detection limit of $20 \mathrm{nM}$. Liu and Lin fabricated an amperometric biosensor based on LBL selfassembling AChE on CNTs-modified GCE and integrated it within a flow injection-detection system. It was highly sensitive for organophosphate pesticides and nerve agents and showed a good precision, reproducibility, and stability [131]. CNTs play a dual significant role in this structure. It provides a robust immobilization sites for a suitable microenvironment to retain the enzyme activity and as a transducer, which amplifies the electrochemical signal of the product of the enzymatic reaction. Rahman et al. fabricated an amperometric lactate biosensor based on MWCNTs and conducting polymer (CP) by covalently immobilizing the lactate dehydrogenase and NADH onto the MWCNTs/CP assembly [132]. The MWCNTs/CP nanocomposite assembly was obtained through the electrochemical polymerization of monomer containing MWCNTs. The analytical results such as sensitivity, selectivity, and stability were found to be improved significantly using MWCNTs/ CP nanocomposite assembly.

The detection of DNA is currently an area of tremendous interest in genetics, clinics, pathology, criminology, pharmacogenetics, food safety, and many other fields. Most of DNA biosensors are developed based on the immobilization of single-stranded DNA onto the electrode surface labeled with an electrochemical indicator to recognize its complementary target sequence. CNTs are promising materials for the development of electrochemical DNA hybridization biosensors. The unique properties of CNTs can be united with the specific molecular-recognition features of DNA by coupling SWNTs to peptide nucleic acid and hybridizing these macromolecular wires with complementary DNA [133]. Both covalent and non-covalent linkage of DNA with CNTs have been reported where the former provide the best stability, accessibility, and selectivity during competitive hybridization [134]. Figure 4 shows an overview of the covalent attachment process. By this attachment, it was found that DNA molecules are accessible to hybridization and strongly favor hybridization with molecules having complementary sequences compared with noncomplementary sequences. The integration of CNTs with other materials has been also used for the immobilization of DNA. Yang et al. described a sensitive DNA hybridization biosensor based on $\mathrm{ZrO}_{2}$ nanoparticles and MWCNTs [135]. The MWCNTs/nano $\mathrm{ZrO}_{2} / \mathrm{CS}$-modified GCE was fabricated by dispersing $\mathrm{ZrO}_{2}$ nanoparticles and MWCNTs in CS and oligonucleotides were immobilized on a modified GCE. The hybridization reaction on the electrode was monitored by DPV analysis where electroactive daunomycin was used as an indicator. Jiao and co-workers applied the same approach for DNA biosensor using $\mathrm{ZnO}$ nanoparticles instead of $\mathrm{ZrO}_{2}$ nanoparticles [136]. Recently, Ma et al. fabricated an electrochemical DNA biosensor based on LBL self-assembly of MWCNTs and GNPs via covalent-bonding interaction [137]. Doxorubicin was used as an intercalator and the hybridization events were monitored electrochemically by DPV measurement. The biosensor showed an improved sensitivity with an excellent reproducibility due to the high catalytic activities of GNPs and the ability of CNTs to promote electron-transfer reactions. A wide linear response range from 0.5 to $0.01 \mathrm{nM}$ with a detection limit of $7.5 \mathrm{pM}$ for target DNA was achieved. Recently, Niu et al. used manganese complex of rutin as a redox intercalator with carboxylic acid group-functionalized MWCNTs and fabricated DNA 
biosensor for DNA hybridization detection [138]. The modified electrode dramatically increased the amount of DNA attachment and the sensitivity of the complementary ssDNA detection mostly due to the large surface area and good charge-transport characteristics of CNTs. Erdem et al. described a new DNA biosensor based on the enhancement of guanine signal at MWCNTs-modified pencil graphite electrode (PGE) using DPV [139]. PGE behaved as a microelectrode array coupled with its higher porosity and showed improved performance compared to GCE. Another new DNA biosensor based on electrochemical impedance was described by Fang's group [140]. They modified GCE using a composite material of PPy and carboxylic group-terminated MWCNTs. A probe with an amino grouptermination was linked onto the PPy/MWCNTs-COOH/GCE by using EDAC and it was found that the hybridization reaction with its complementary decreased the electron transfer resistance. Zhang's group also used PPy and MWCNTs to develop a high sensitive and selective biosensor for DNA hybridization based on the immobilization of DNA probe within electropolymerized PPy on a MWCNTs paste electrode [141]. Ethidium bromide (EB) was used as an intercalator and the current change generated from it was monitored. Only the complementary DNA, compared to the five-point mismatched and non-complementary sequences, gave an obvious current flow and a detection limit of $0.85 \mathrm{pM}$ was obtained. An electrochemical DNA biosensor based on palladium nanoparticles combined with MWCNTs was suggested by Chang et al. [142]. MB was used as an indicator and the hybridization was monitored by DPV measurement. A lower detection limit of $120 \mathrm{fM}$ for the target DNA was achieved and the improved sensitivity was attributed to the ability of CNTs promoting electron-transfer process and the high catalytic activities of palladium nanoparticles for electrochemical reaction of MB.

Electrochemical immunosensors are the popular area of study due to their high specificity, sensitivity, and stability. Most of approaches are based on an enzyme-linked immunosorbent assay (ELISA) system built on electrode surfaces. The amount of enzyme-linked antigen bound to the immobilized antibody is determined by the relative concentration of the free and conjugated antigen and quantified by the rate of enzymic reaction. To avoid the additional steps for labeling, the label-free immunosensors are getting much interest. CNTs are potential materials for the fabrication of labeled or label-free immunosensors. The carboxylic acid groups-functionalized CNTs can be used as an antibody immobilizing platform. CNTs can also be used in the detection probe of an immunosensor. Yun et al. developed a label-free immunosensor based on CNTs array electrodes for direct electrochemical detection of antigen-antibody binding reaction [143]. Anti-mouse IgG was covalently immobilized on the carboxylic acid-terminated nanotube array and the binding was characterized by $\mathrm{CV}$ and electrochemical impedance spectroscopy (EIS). The detection limit was found to be 200 $\mathrm{ng} / \mathrm{mL}$. EIS was chosen here as the analytical approach because of its ability to analyze the electrochemical response of the electrode over a wide frequency range. Electrodes modified with SWCNTs array have also been used by Okuno et al. to fabricate a label-free electrochemical immunosensor for prostate-specific antigen [144]. Yu et al. described an electrochemical immunosensor using SWCNTs forest platforms with multi-label secondary antibody-nanotube bioconjugates for highly sensitive detection of a cancer biomarker in serum and tissue lysates [145]. A great amplification of the sensitivity was achieved by using bioconjugates featuring HRP labels and secondary antibodies (Ab2) linked to MWCNTs at high HRP/Ab2 ratio (Figure 5). A low detection 
limit of $4 \mathrm{pg} / \mathrm{mL}$ for prostate specific antigen was obtained in $10 \mu \mathrm{L}$ undiluted calf serum with this strategy.

Figure 4. Overall scheme for fabrication of covalently linked DNA-nanotube adducts. Reprinted with permission from [134]. Copyright (2002) American Chemical Society.
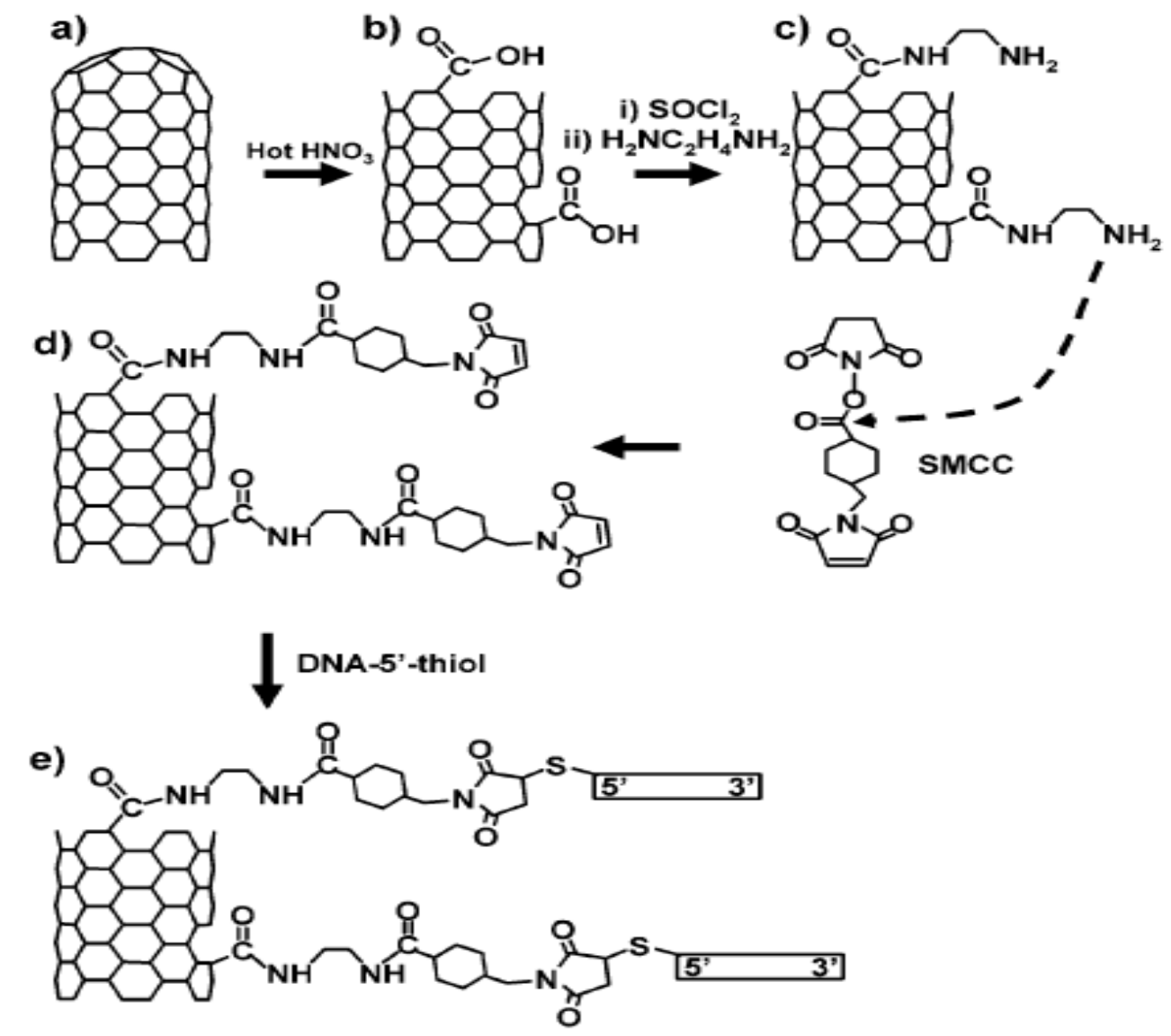

Figure 5. Configuration of the nanotube immunosensor. Reprinted with permission from [145]. Copyright (2006) American Chemical Society.

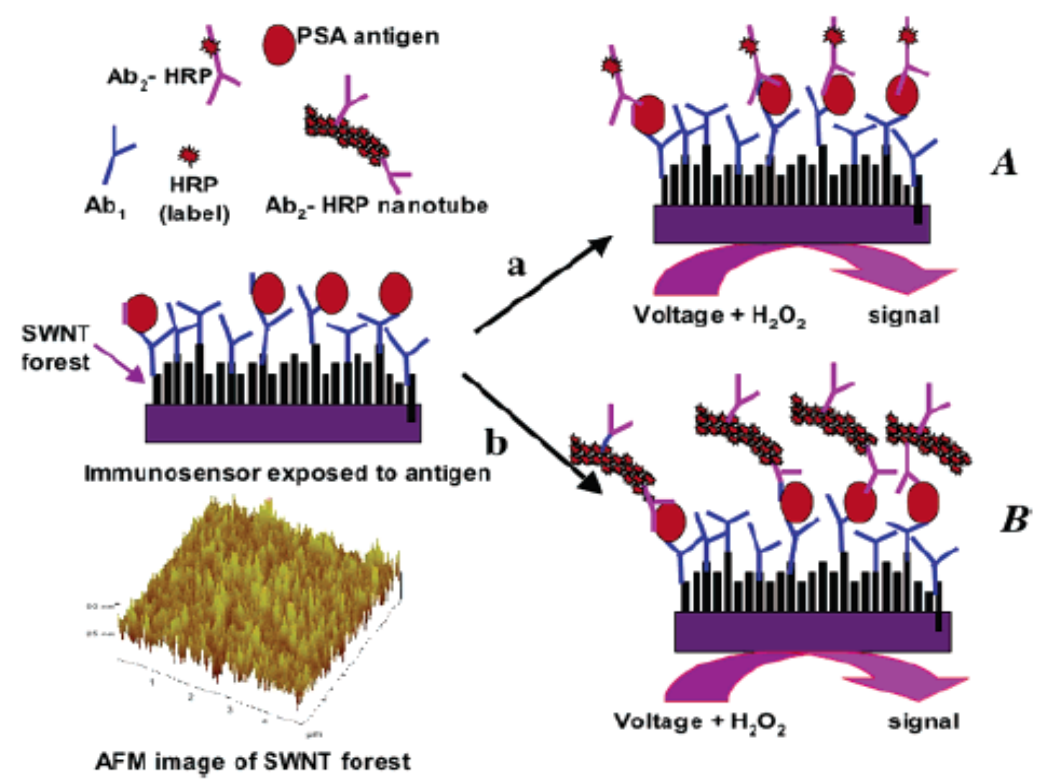


Polymer-CNTs composite films have been widely used for the fabrication of electrochemical immunosensors. Cataldo et al. evaluated an amperometric immunosensor based on the covalently bound anti-biotin antibodies (Ab) embedded into a polylysine (PLL)-SWCNTs composite layer [146]. An improved amperometric detection limit of $10 \mathrm{pM}$ was obtained for biotin (Ag) labeled with HRP by incorporating SWCNTs into PLL-antibody assemblies. One advantage of this proposed immunosensor is the improved thermal stability of the anti-biotin embedded into the PLL matrix. Poly (3,4-ethylene-dioxythiophene)-coated MWCNTs have been used for the first time as a voltammetric sensor in an immunoassay for Cholera Toxin (CT) by Ho's group [147]. Ganglioside (GM1)functionalized liposomes encapsulated with potassium ferrocyanide (an electroactive redox marker) were used for the detection of CT. A wide linear range from $10 \mathrm{fg} / \mathrm{mL}$ to $0.1 \mu \mathrm{g} / \mathrm{mL}$ with a detection limit of $0.1 \mathrm{fg} / \mathrm{mL}$ of CT was achieved. Sánchez et al. described an electrochemical immunosensor method based on polysulfone membrane encapsulating MWCNTs and immunoreagents layered on disposable screen- printed electrodes [148]. The immunocomposite acts both as reservoir of immunological material and transducer while offering high surface area, high toughness, and mechanical flexibility.

Chitosan (CS), a biological cationic macromolecule, provides a suitable environment for biomolecules to keep their activity. Their combination with highly conductive CNTs as an immunosensing platform has attracted considerable interest. Recently, Li et al. proposed a novel amperometric immunosensor for human chorionic gonadotropin (HCG) assay incorporating $\mathrm{Tb}$ and $\mathrm{Hb}$ on the MWCNTs-CS modified GCE, followed by electrostatic adsorption of a conducting GNPs film as sensing interface [149]. The MWCNTs-CS matrix provided a congenial microenvironment for the immobilization of biomolecules and promoted the electron transfer of the redox active species, thus, enhanced the sensitivity of the immunosensor. CS can be also used for the development of a highly sensitive and label-free amperometric immunosensor for carcinoembryonic antigen detection [150]. The fabrication of the immunosensor was based on LBL assembly of GNPs, MWCNTs-thionine, and CS on 3-mercaptopropanesulfonic acid (MPS)-modified gold electrode surface. The detection limit of carcinoembryonic antigen with this label-free amperometric immunosensor was determined to be 0.01 $\mathrm{ng} / \mathrm{ml}$.

The practical application of CNTs can be found in a very limited area only. It is partially attributable to the lack of simple modification skill of electrode surfaces with CNTs. To overcome this obstacle, Yang and coworkers developed two different methods for the modification of ITO electrode with CNTs. The ITO electrodes are advantageous to use in optical experiment and to make electrochemical (bio) sensors because it is optically transparent and display high conductivity with low background current. Initially, they fabricated thin film of MWCNTs by simply physisorbing of CNTs on the ITO electrode from the aqueous solution of carboxylated MWCNTs [151]. To make thin film of CNTs on ITO electrode, they covalently immobilized the carboxylated SWCNTs on an aminefunctionalized ITO surface using dicyclohexylcarbodiimide as a coupling agent [152]. Both films showed low background currents and good electrocatalytic properties toward the oxidation of $p$ aminophenol ( $p$-AP), which is an enzymatic product of enzyme substrate ( $p$-aminophenyl phosphate). Both thin CNT films could be used to fabricate sandwich-type immunosensors because of the good electrocatlytic property with a low background current, and the ability of immobilization of biomolecules on the sidewall of the CNTs. To fabricate the sandwich type immunosensor, avidin was 
immobilized first on the hydrophobic sidewalls of CNTs to capture biotinylated anti-mouse IgG. Then, the target mouse IgG was sandwiched between the biotinylated anti-mouse IgG and alkaline phosphatase-conjugated antimouse IgG. The alkaline phosphatase catalyzed the conversion of electroactive $p$-AP from non electroactive $p$-aminophenyl phosphate. The enhancement of the generation of $p$-AP upon increase of the concentration of target mouse IgG resulted in the strength of the signal. The thin films of MWCNTs and SWCNTs gave the detection limits of $10 \mathrm{pg} / \mathrm{mL}$ and 100 $\mathrm{pg} / \mathrm{mL}$, respectively. They are comparable to those of sensors with a commonly employed enzymelinked immunosorbent assay (pM range).

\section{Application of CNTs-Based Sensors to Real Sample Analysis}

CNTs-based sensors can be applied in real sample analysis in different areas such as biomedical, food, agriculture, and fishing industries. There are many biomedical sensing applications where CNTsbased sensors perform better in real sample analysis. CNTs-based sensors can be used in commercial food samples to detect undesired chemical residues resulting from animal drugs, food additives, pesticides, and other environmental contaminants in raw and processed foods. Rahman et al. determined the lactate concentration in commercial milk using CNTs-based electrochemical biosensor and compared the result with that obtained from a biochemical analyzer [132]. The biosensor result was found to be in good agreement with the result from biochemical analyzer $(0.18 \pm 0.006 \mathrm{mM}$ and $0.174 \pm 0.01 \mathrm{mM}$, respectively). CNTs based electrochemical sensors are also widely used in real blood and urine samples analyses. Table 1 summarizes the applications of some CNTs-based electrochemical sensors to real sample analyses.

Table 1. Applications of CNTs-based electrochemical sensors in real samples.

\begin{tabular}{llllc}
\hline \multicolumn{1}{c}{ Electrode } & Analyte & Real sample & $\begin{array}{c}\text { Detection } \\
\text { limit }\end{array}$ & Reference \\
\hline GCE/P3MT/SWNTs/Nafion & dopamine & serum & $5.00 \mathrm{nM}$ & {$[23]$} \\
GCE/ PANI /MWNTs / $\beta$-CD & dopamine & injection & $12.0 \mathrm{nM}$ & {$[25]$} \\
Au/SWNTs & rutin & tablet (drug) & $10.0 \mathrm{nM}$ & {$[54]$} \\
GCE/ MWNTs / $\beta$-CD & rutin & urine & $0.20 \mu \mathrm{M}$ & {$[55]$} \\
GCE/ MWNTs / PtNC & estrogen & serum & $0.18 \mu \mathrm{M}$ & {$[60]$} \\
$\mathrm{CPE} / \mathrm{MWCNTs} / \mathrm{CoSal}$ & tryptophan & serum & $0.10 \mu \mathrm{M}$ & {$[64]$} \\
GCE /SWNTs /BMIMPF 6 & methylparathion & lake water /apple & $1.00 \mathrm{nM}$ & {$[67]$} \\
GCE /SWNTs /BMIMPF 6 & xanthine & serum / urine & $2.00 \mathrm{nM}$ & {$[68]$} \\
GCE/ MWNTs / poly-ACBK & dihydroxybenzene & water & $0.10 \mu \mathrm{M}$ & {$[73]$} \\
\hline
\end{tabular}


Table 1. Cont.

\begin{tabular}{|c|c|c|c|c|}
\hline GCE/ MWNTs & noscapine & drug/blood & $80.0 \mathrm{nM}$ & {$[79]$} \\
\hline GCE/ MWNTs & captopril & drug/urine & $0.20 \mu \mathrm{M}$ & {$[80]$} \\
\hline ITO/MWCNTs/GOx/NFE & glucose & serum & $1.00 \mu \mathrm{M}$ & {$[96]$} \\
\hline Teflon/MWCNTs/Au $\mathrm{Aull}_{\text {col }} / \mathrm{GOx}$ & glucose & beverage & $17.0 \mu \mathrm{M}$ & {$[98]$} \\
\hline Teflon/MWCNTs/Au $\mathrm{col}_{\mathrm{col}} / \mathrm{ADH}$ & ethanol & beer & $4.70 \mu \mathrm{M}$ & {$[99]$} \\
\hline GCE/CS/CNTs/ Au-PtNPs & glucose & blood/urine & $0.20 \mu \mathrm{M}$ & [103] \\
\hline GCE/MWNTs/ FMC-BSA & hydrogen peroxide & milk & $0.20 \mu \mathrm{M}$ & [111] \\
\hline CPE/MB/MWCNTs/ADH & ethanol & beverage & $5.00 \mu \mathrm{M}$ & [118] \\
\hline $\mathrm{Pt} / \mathrm{MWCNTs} / \mathrm{ChOx}$ & choline & serum & $0.10 \mu \mathrm{M}$ & [124] \\
\hline Au/pTTCA/MWNTs/LDH & lactate & milk/serum & $1.00 \mu \mathrm{M}$ & [132] \\
\hline \multicolumn{5}{|c|}{$\begin{array}{l}\text { P3MT, poly(3-methylthiophene); PANI, polyaniline; } \beta \text {-CD, } \beta \text {-cyclodextrin; ABSA, aminobenzene } \\
\text { sulphonic acid; PtNC, Platinum nano-clusters; CPE, carbon paste electrode; CoSal, cobalt salophen; } \\
\text { BMIMPF }_{6} \text { 1-butyl-3-methylimidazolium hexafluophosphate; ACBK, acid chrome blue K; ADH, alcohol } \\
\text { dehydrogenase; CS, chitosan; FMC-BSA, ferrocene monocarboxylic acid-bovine serum albumin; ChOx, } \\
\text { choline oxidase; pTTCA, poly-5,2'-5',2''-terthiophene-3'-carboxylic acid; LDH, Lactate dehydrogenase }\end{array}$} \\
\hline
\end{tabular}

\section{Conclusions}

CNTs are now used extensively in the fabrication of novel nanostructured electrochemical sensors. CNTs-modified electrodes have many advantages over other forms of carbon electrodes due to their small size, high electrical and thermal conductivity, high chemical stability, high mechanical strength, and high specific surface area. Their small diameter and long length allow them to be plugged into proteins with better electro-activity compared to other carbon based electrodes. The promoted electron transfer and direct electrochemistry of proteins at CNTs-based electrochemical sensing films are now well documented. Due to its faster electron transfer over other carbon based materials, CNTs show excellent electrocatalytic activity in redox behavior of different compounds. Analytical sensing at CNTs-modified electrodes results in low detection limits, high sensitivities, reduction of overpotentials, and resistance to surface fouling. The aforementioned outstanding properties of CNTs make them an exciting alternative for the development of novel electrochemical sensors and biosensors. However, there are a number of challenges to be addressed to fulfill the application of CNTs for sensors. The commercial production of pure and defect-free CNTs is difficult and costly. Processing of CNTs is still not fully controlled. For example, aggregations of tubes are not prevented, lengths are not uniformly obtainable, and non-specific adsorptions of proteins to the walls of nanotubes are not prevented. CNTs can cause health risks due to their toxicity and harmful effects in the lung, where they can agglomerate leading to suffocation. The toxicological impact of nanotubes is an obstacle for the application of nanotubes in bioelectronics and any subject integrated with living biological systems. Another limitation is that CNTs are commonly insoluble in most solvents, which has greatly hindered their promising practical applications. Covalent and non-covalent functionalizations of CNTs are not very 
effective in overcoming this limitation. CNTs can be dispersed in Nafion, Teflon, CS, mineral oils, solgel silica, and in some polymer but these methods possibly impair the chemical properties of CNTs or decrease their conductivity. While there have some disadvantages of CNT-modified electrode for sensing application, the continuous growing research interest in this field is contributing to overcome them. It is believed that the merits of CNT-based sensors will bring dramatic changes to future sensor industry.

\section{Acknowledgements}

This work was supported by the Regional Innovation Center Program of the Ministry of Knowledge Economy through the Bio-Food \& Drug Research Center at Konkuk University, Korea.

\section{References}

1. Padigi, S.K.; Reddy, R.K.K.; Prasad, S. Carbon nanotube based aliphatic hydrocarbon sensor. Biosens. Bioelectron. 2007, 22, 829-837.

2. Iijima, S. Helical microtubules of graphitic carbon. Nature 1991, 354, 56-58.

3. Sun, G.; Liu, S.; Hua, K.; Lv, X.; Huang, L.; Wang, Y. Electrochemical chlorine sensor with multi-walled carbon nanotubes as electrocatalysts. Electrochem.Commun. 2007, 9, 2436-2440.

4. Zhu, L.; Yang, R.; Zhai, J.; Tian, C. Bienzymatic glucose biosensor based on co-immobilization of peroxidase and glucose oxidase on a carbon nanotubes electrode. Biosens. Bioelectron. 2007, 23, 528-535.

5. Santhosh, P.; Manesh, K.M.; Gopalan, A.; Lee, K.P. Novel amperometric carbon monoxide sensor based on multi-wall carbon nanotubes grafted with polydiphenylamine-fabrication and performance. Sens. Actuat. B-Chem. 2007, 125, 92-99.

6. Du, P.; Liu, S.; Wu, P.; Cai, C. Preparation and characterization of room temperature ionic liquid/single-walled carbon nanotube nanocomposites and their application to the direct electrochemistry of heme-containing proteins/enzymes. Electrochim. Acta 2007, 52, 6534-6547.

7. Sato, N.; Okuma, H. Development of single-wall carbon nanotubes modified screen-printed electrode using a ferrocene-modified cationic surfactant for amperometric glucose biosensor applications. Sens. Actuat. B-Chem. 2008, 129, 188-194.

8. Banks, C.E.; Compton, R.G. New electrodes for old: from carbon nanotubes to edge plane pyrolytic graphite. Analyst 2006, 131, 15-21.

9. Moore, R.R.; Banks, C.E.; Compton, R.G. Basal plane pyrolytic graphite modified electrodes: comparison of carbon nanotubes and graphite powder as electrocatalysts. Anal. Chem. 2004, 76, 2677-2682.

10. Banks, C.E.; Moore, R.R.; Davies, T.J.; Compton, R.G. Investigation of modified basal plane pyrolytic graphite electrodes:definitive evidence for the electrocatalytic properties of the ends of carbon nanotubes. Chem. Commun. 2004, 1804-1805.

11. Banks, C.E.; Davies, T.J.; Wildgoose, G.G.; Compton, R.G. Electrocatalysis at graphite and carbon nanotube modified electrodes: edge-plane sites and tube ends are the reactive sites. Chem. Commun. 2005, 829-841. 
12. Holloway, A.F.; Wildgoose, G.G.; Compton, R.G.; Shao, L.; Green; M.L.H. The influence of edge-plane defects and oxygen-containing surface groups on the voltammetry of acid-treated, annealed and "super-annealed" multiwalled carbon nanotubes. J. Solid State Electrochem.2008 $12,1337-1348$.

13. Chou, A.; Bo"cking, T.; Singh, N.K.; Gooding, J.J. Demonstration of the importance of oxygenated species at the ends of carbon nanotubes for their favourable electrochemical properties. Chem. Commun. 2005, 842-844.

14. Liu, J.; Chou, A.; Rahmat, W.; Paddon-Row, M.N.; Gooding, J.J. Achieving direct electrical connection to glucose oxidase using aligned single walled carbon nanotube arrays. Electroanal. 2005, 17, 38-46.

15. Gooding, J.J.; Chou, A.; Liu, J.; Losic, D.; Shapter, J.G.; Hibbert, D.B. The effects of the lengths and orientations of single-walled carbon nanotubes on the electrochemistry of nanotube-modified electrodes. Electrochem.Commun. 2007, 9, 1677-1683.

16. Chou, A.; Bo“cking, T.; Liu, R.; Singh, N.K.; Moran, G.; Gooding, J.J. Effect of dialysis on the electrochemical properties of acid-oxidized single-walled carbon nanotubes. J. Phys. Chem. C 2008, 112, 14131-14138.

17. Pumera, M. Carbon nanotubes contain residual metal catalyst nanoparticles even after washing with nitric acid at elevated temperature because these metal nanoparticles are sheathed by several graphene sheets. Langmuir 2007, 23, 6453-6458.

18. Kolodiazhnyi, T.; Pumera, M. Towards an ultrasensitive method for the determination of metal impurities in carbon nanotubes. Small 2008, 9, 1476-1484.

19. Gong, K.; Du, F.; Xia, Z.; Durstock, M.; Dai, L. Nitrogen-doped carbon nanotube arrays with high electrocatalytic activity for oxygen reduction. Science 2009, 323, 760-764.

20. Lawrence, N.S.; Deo, R.P.; Wang, J. Comparison of the electrochemical reactivity of electrodes modified with carbon nanotubes from different sources. Electroanalysis 2005, 17, 65-72.

21. Musameh, M.; Lawrence, N.S.; Wang, J. Electrochemical activation of carbon nanotubes. Electrochem.Commun. 2005, 7, 14-18.

22. Rahman, M.A.; Kumar, P.; Park, D.S.; Shim, Y.B. Electrochemical sensors based on organic conjugated polymers. Sensors 2008, 8, 118-141.

23. Wang, H.S.; Li, T.H.; Jia, W.L.; Xu, H.Y. Highly selective and sensitive determination of dopamine using a Nafion/carbon nanotubes coated poly (3-methylthiophene) modified electrode. Biosens. Bioelectron. 2006, 22, 664-669.

24. Zhang, Y.; Cai, Y.; Su, S. Determination of dopamine in the presence of ascorbic acid by poly (styrene sulfonic acid) sodium salt/single-wall carbon nanotube modified glassy carbon electrode. Anal. Biochem. 2006, 350, 285-291.

25. Yin, T.; Wei, W.; Zeng, J. Selective detection of dopamine in the presence of ascorbic acid by use of glassy-carbon electrodes modified with both polyaniline film and multi-walled carbon nanotubes with incorporated $\beta$-cyclodextrin. Anal. Bioanal. Chem. 2006, 386, 2087-2094.

26. Angeles, G.A.; Lo'pez, B.P.; Pardave, M.P.; Silva, M.T.R.; Alegret, S.; Merkoci, A. Enhanced host-guest electrochemical recognition of dopamine using cyclodextrin in the presence of carbon nanotubes. Carbon 2008, 46, 898-906. 
27. Li, Y.; Wang, P.; Wang, L.; Lin, X. Overoxidized polypyrrole film directed single-walled carbon nanotubes immobilization on glassy carbon electrode and its sensing applications. Biosens. Bioelectron. 2007, 22, 3120-3125.

28. Liu, A.; Honma, I.; Zhou, H. Simultaneous voltammetric detection of dopamine and uric acid at their physiological level in the presence of ascorbic acid using poly (acrylic acid)-multiwalled carbon-nanotube composite-covered glassy-carbon electrode. Biosens. Bioelectron. 2007, 23, 7480.

29. Yogeswaran, U.; Chen, S.M. Separation and concentration effect of f-MWCNTs on electrocatalytic responses of ascorbic acid, dopamine and uric acid at f-MWCNTs incorporated with poly (neutral red) composite films. Electrochim. Acta 2007, 52, 5985-5996.

30. Yogeswaran, U.; Thiagarajan, S.; Chen, S.M. Nanocomposite of functionalized multiwall carbon nanotubes with nafion, nano platinum, and nano gold biosensing film for simultaneous determination of ascorbic acid, epinephrine, and uric acid. Anal. Biochem. 2007, 365, 122-131.

31. Yi, H.; Zheng, D.; Hu, C.; Hu, S. Functionalized multiwalled carbon nanotubes through in situ electropolymerization of brilliant cresyl blue for determination of epinephrine. Electroanalysis 2008, 20, 1143-1146.

32. Valentini, F.; Palleschi, G.; Morales, E.L.; Orlanducci, S.; Tamburri, E.; Terranova, M.L. Functionalized single-walled carbon nanotubes modified microsensors for the selective response of epinephrine in presence of ascorbic acid. Electroanal. 2007, 19, 859-869.

33. Zhai, X.; Wei, W.; Zeng, J.; Gong, S.; Yin, J. Layer-by-layer assembled film based on chitosan/carbon nanotubes, and its application to electrocatalytic oxidation of NADH. Microchim. Acta 2006, 154, 315-320.

34. Wang, Q.; Tang, H.; Xie, Q.; Tan, L.; Zhang, Y.; Li, B.; Yao, S. Room-temperature ionic liquids/multi-walled carbon nanotubes/chitosan composite electrode for electrochemical analysis of NADH. Electrochim. Acta, 2007, 52, 6630-6637.

35. Radoi, A.; Compagnone, D.; Valcarcel, M.A.; Placidi, P.; Materazzi, S.; Moscone, D.; Palleschi, G. Detection of NADH via electrocatalytic oxidation at single-walled carbon nanotubes modified with Variamine blue. Electrochim. Acta 2008, 53, 2161-2169.

36. Liu, A.; Watanabe, T.; Honma, I.; Wang, J.; Zhou, H. Effect of solution pH and ionic strength on the stability of poly(acrylic acid)-encapsulated multiwalled carbon nanotubes aqueous dispersion and its application for NADH sensor. Biosens. Bioelectron. 2006, 22, 694-699.

37. Zeng, J.; Gao, X.; Wei, W.; Zhai, X.; Yin, J.; Wu, L.; Liu, X.; Liu, K.; Gong, S. Fabrication of carbon nanotubes/poly(1,2-diaminobenzene) nanoporous composite via multipulse chronoamperometric electropolymerization process and its electrocatalytic property toward oxidation of NADH. Sens. Actuat. B-Chem. 2007, 120, 595-602.

38. Tu, X.; Xie, Q.; Huang, Z.; Yang, Q.; Yao, S. Synthesis and characterization of novel quinoneamine polymer/carbon nanotubes composite for sensitive electrocatalytic detection of NADH. Electroanalysis 2007, 19, 1815-1821.

39. Ag"u'1, L.; Farfal, C.P.; Sedẽno, P.Y.; Pingarr'on, J.M. Poly-(3-methylthiophene)/carbon nanotubes hybrid composite-modified electrodes. Electrochim. Acta 2007, 52, 7946-7952. 
40. Tkac, J.; Ruzgas, T. Dispersion of single walled carbon nanotubes. Comparison of different dispersing strategies for preparation of modified electrodes toward hydrogen peroxide detection. Electrochem.Commun. 2006, 8, 899-903.

41. Sun, N.; Guan, L.; Shi, Z.; Li, N.; Gu, Z.; Zhu, Z.; Li, M.; Shao, Y. Ferrocene peapod modified electrodes: preparation, characterization, and mediation of $\mathrm{H}_{2} \mathrm{O}_{2}$. Anal. Chem. 2006, 78, 60506057.

42. Yuan, S.; He, Q.; Yao, S.; Hu, S. Mercury-free detection of europium (III) at a glassy carbon electrode modified with carbon nanotubes by adsorptive stripping voltammetry. Anal. Lett. 2006, 39, 373-385.

43. Suna, D.; Xie, X.; Cai, Y.; Zhang, H.; Wu, K. Voltammetric determination of $\mathrm{Cd}^{2+}$ based on the bifunctionality of single-walled carbon nanotubes-Nafion film. Anal. Chim. Acta 2007, 581, $27-$ 31.

44. Profumo, A.; Fagnoni, M.; Merli, D.; Quartarone, E.; Protti, S.; Dondi, D.; Albini, A. Multiwalled carbon nanotube chemically modified gold electrode for inorganic as speciation and Bi(III) determination. Anal. Chem. 2006, 78, 4194-4199.

45. Jo, S.; Jeong, H.; Bae, S.R.; Jeon, S. Modified platinum electrode with phytic acid and singlewalled carbon nanotube: Application to the selective determination of dopamine in the presence of ascorbic and uric acids. Microchem. J. 2008, 88, 1-6.

46. Li, Z.; Chen, J.; Pan, D.; Tao, W.; Nie, L.; Yao, S. A sensitive amperometric bromate sensor based on multi-walled carbon nanotubes/phosphomolybdic acid composite film. Electrochim. Acta 2006, 51, 4255-4261.

47. Wu, Y.; Ye, S.; Hu, S. Electrochemical study of lincomycin on a multi-wall carbon nanotubes modified glassy carbon electrode and its determination in tablets. J. Pharm. Biomed. Anal. 2006, $41,820-824$.

48. Ming, L.; Xi, X.; Chen, T.; Liu, J. Electrochemical determination of trace sudan I contamination in chili powder at carbon nanotube modified electrodes. Sensors 2008, 8, 1890-1900.

49. Wang, F.; Chen, L.; Chen, X.; Hu, S. Studies on electrochemical behaviors of acyclovir and its voltammetric determination with nano-structured film electrode. Anal. Chim. Acta 2006, 576, $17-$ 22.

50. Li, C. Voltammetric determination of 2-chlorophenol using a glassy carbon electrode coated with multi-wall carbon nanotube-dicetyl phosphate film. Microchim. Acta 2007, 157, 21-26.

51. Huang, K.J.; Luo, D.F.; Xie, W.Z.; Yu, Y.S. Sensitive voltammetric determination of tyrosine using multi-walled carbon nanotubes/4-aminobenzeresulfonic acid film-coated glassy carbon electrode. Colloid Surf. B 2008, 61, 176-181.

52. Wu, K.; Wang, H.; Chen, F.; Hu, S. Electrochemistry and voltammetry of procaine using a carbon nanotube film coated electrode. Bioelectrochemistry 2006, 68, 144-149.

53. Zhua, Y.; Zhang, Z.; Zhao, W.; Pang, D. Voltammetric behavior and determination of phenylephrine at a glassy carbon electrode modified with multi-wall carbon nanotubes. Sens. Actuat. B-Chem. 2006, 119, 308-314.

54. Zeng, B.; Wei, S.; Xiao, F.; Zhao, F. Voltammetric behavior and determination of rutin at a single-walled carbon nanotubes modified gold electrode. Sens. Actuat. B-Chem. 2006, 115, 240 246. 
55. He, J.L.; Yang, Y.; Yang, X.; Liu, Y.L.; Liu, Z.H.; Shen, G.L.; Yu, R.Q. ß-Cyclodextrin incorporated carbon nanotube-modified electrode as an electrochemical sensor for rutin. Sens. Actuat. B-Chem. 2006, 114, 94-100.

56. Wang, G.; Hu, N.; Wang, W.; Li, P.; Gu, H.; Fang, B. Preparation of carbon nanotubes/neutral red composite film modified electrode and its catalysis on rutin. Electroanalysis 2007, 19, 2329-2334.

57. Zare, H.R.; Sobhani, Z.; Ardakani, M.M. Electrocatalytic oxidation of hydroxylamine at a rutin multi-wall carbon nanotubes modified glassy carbon electrode: Improvement of the catalytic activity. Sens. Actuat. B-Chem. 2007, 126, 641-647.

58. Hrapovic, S.; Majid, E.; Liu, Y.; Male, K.; Luong, J.H.T. Metallic nanoparticle-carbon nanotube composites for electrochemical determination of explosive nitroaromatic compounds. Anal. Chem. 2006, 78, 5504-5512.

59. Xiao, F.; Zhao, F.; Li, J.; Yan, R.; Yu, J.; Zeng, B. Sensitive voltammetric determination of chloramphenicol by using single-wall carbon nanotube-gold nanoparticle-ionic liquid composite film modified glassy carbon electrodes. Anal. Chim. Acta 2007, 596, 79-85.

60. Lin, X.; Li, Y. A sensitive determination of estrogens with a Pt nano-clusters/multi-walled carbon nanotubes modified glassy carbon electrode. Biosens. Bioelectron. 2006, 22, 253-259.

61. Yang, P.; Wei, W.; Tao, C. Determination of trace thiocyanate with nano-silver coated multiwalled carbon nanotubes modified glassy carbon electrode. Anal. Chim. Acta 2007, 585, 331-336.

62. Liu, H.; Wang, G.; Chen, D.; Zhang, W.; Li, C.; Fang, B. Fabrication of polythionine/NPAu/MWNTs modified electrode for simultaneous determination of adenine and guanine in DNA. Sens. Actuat. B-Chem. 2008, 128, 414-421.

63. Zhuang, Q.; Chen, J.; Chen, J.; Lin, X. Electrocatalytical properties of bergenin on a multi-wall carbon nanotubes modified carbon paste electrode and its determination in tablets. Sens. Actuat. B-Chem. 2008, 128, 500-506.

64. Shahrokhian, S.; Fotouhi, L. Carbon paste electrode incorporating multi-walled carbon nanotube/cobalt salophen for sensitive voltammetric determination of tryptophan. Sens. Actuat. BChem. 2007, 123, 942-949.

65. Shahrokhian, S.; Mehrjardi, H.R.Z. Simultaneous voltammetric determination of uric acid and ascorbic acid using a carbon-paste electrode modified with multi-walled carbon nanotubes/nafion and cobalt (II)-nitrosalophen. Electroanalysis 2007, 19, 2234-2242.

66. Shahrokhian, S.; Mehrjardi, H.R.Z. Application of thionine-nafion supported on multi-walled carbon nanotube for preparation of a modified electrode in simultaneous voltammetric detection of dopamine and ascorbic acid. Electrochim. Acta 2007, 52, 6310-6317.

67. Fan, S.; Xiao, F.; Liu, L.; Zhao, F.; Zeng, B. Sensitive voltammetric response of methylparathion on single-walled carbon nanotube paste coated electrodes using ionic liquid as binder. Sens. Actuat. B-Chem. 2008, 132, 34-39.

68. Xiao, F.; Ruan, C.; Li, J.; Liu, L.; Zhao, F.; Zeng, B. Voltammetric determination of xanthine with a single-walled carbon nanotube-ionic liquid paste modified glassy carbon electrode. Electroanalysis. 2008, 20, 361-366.

69. Yan, Q.; Zhao, F.; Li, G.; Zeng, B. Voltammetric determination of uric acid with a glassy carbon electrode coated by paste of multiwalled carbon nanotubes and ionic liquid. Electroanalysis 2006, $18,1075-1080$. 
70. Abbaspour, A.; Mirzajani, R. Electrochemical monitoring of piroxicam in different pharmaceutical forms with multi-walled carbon nanotubes paste electrode. J. Pharm. Biomed. Anal. 2007, 44, 41-48.

71. Li, Z.; Junfeng, S. Voltammetric behavior of urapidil and its determination at multi-wall carbon nanotube paste electrode. Talanta 2007, 73, 943-947.

72. Xiao, P.; Zhao, F.; Zeng, B. Voltammetric determination of quercetin at a multi-walled carbon nanotubes paste electrode. Microchem. J. 2007, 85, 244-249.

73. Yang, P.; Wei1, W.; Yang, L. Simultaneous voltammetric determination of dihydroxybenzene isomers using a poly (acid chrome blue K)/carbon nanotube composite electrode. Microchim. Acta 2007, 157, 229-235.

74. Wang, C.; Mao, Y.; Wang, D.; Yang, G.; Qu, Q.; Hu, X. Voltammetric determination of terbinafine in biological fluid at glassy carbon electrode modified by cysteic acid/carbon nanotubes composite film. Bioelectrochemistry 2008, 72, 107-115.

75. Zhu, L.; Tian, C.; Zhai, J.; Yang, R. Sol-gel derived carbon nanotubes ceramic composite electrodes for electrochemical sensing. Sens. Actuat. B-Chem. 2007, 125, 254-261.

76. Wang, J.; Tangkuaram, T.; Loyprasert, S.; Alvarez, T.V.; Veerasai, W.; Kanatharana, P.; Thavarungkul, P. Electrocatalytic detection of insulin at RuOx/carbon nanotube-modified carbon electrodes. Anal. Chim. Acta 2007, 581, 1-6.

77. Snider, R. M.; Ciobanu, M.; Rue, A.E.; Cliffel, D.E. A multiwalled carbon nanotube/dihydropyran composite film electrode for insulin detection in a microphysiometer chamber. Anal. Chim. Acta 2008, 609, 44-52.

78. Vega, D.; Agüí, L.; Cortés, A.G.; Sedeño, P.Y.; Pingarrón, J. M. Voltammetry and amperometric detection of tetracyclines at multi-wall carbon nanotube modified electrodes. Anal. Bioanal. Chem. 2007, 389, 951-958.

79. Rezaei, B.; Zare, S.Z.M. Modified glassy carbon electrode with multiwall carbon nanotubes as a voltammetric sensor for determination of noscapine in biological and pharmaceutical samples. Sens. Actuat. B-Chem. 2008, 134, 292-299.

80. Rezaei, B.; Damiri, S. Voltammetric behavior of multi-walled carbon nanotubes modified electrode-hexacyanoferrate (II) electrocatalyst system as a sensor for determination of captopril. Sens. Actuat. B-Chem. 2008, 134, 324-331.

81. Buratti, S.; Brunetti, B.; Mannino, S. Amperometric detection of carbohydrates and thiols by using a glassy carbon electrode coated with Co oxide/multi-wall carbon nanotubes catalytic system. Talanta 2008, 76, 454-457.

82. Wang, J. Electrochemical glucose biosensors. Chem. Rev. 2008, 108, 814-825.

83. Heller, A.; Feldman, B. Electrochemical glucose sensors and their applications in diabetes management. Chem. Rev. 2008, 108, 2482-2505.

84. Tsai, Y.C.; Li, S.C.; Liao, S.W. Electrodeposition of polypyrrole-multiwalled carbon nanotubeglucose oxidase nanobiocomposite film for the detection of glucose. Biosens. Bioelectron. 2006, 22, 495-500.

85. Huang, J.; Yang, Y.; Shi, H.; Song, Z.; Zhao, Z.; Anzai, J.; Osa, T.; Chen, Q. Multi-walled carbon nanotubes-based glucose biosensor prepared by a layer-by-layer technique. Mater. Sci. Eng. C 2006, 26, 113-117. 
86. Liu, G.; Lin, Y. Amperometric glucose biosensor based on self-assembling glucose oxidase on carbon nanotubes. Electrochem.Commun. 2006, 8, 251-256.

87. Zhao, H.; Ju, H. Multilayer membranes for glucose biosensing via layer-by-layer assembly of multiwall carbon nanotubes and glucose oxidase. Anal. Biochem. 2006, 350, 138-144.

88. Liu, Y.; Wu, S.; Ju, H.; Xu, Li. Amperometric glucose biosensing of gold nanoparticles and carbon nanotube multilayer membranes. Electroanalysis 2007, 19, 986-992.

89. Xu, L.; Zhu, Y.; Tang, L.; Yang, X.; Li, C. Biosensor based on self-assembling glucose oxidase and dendrimer-encapsulated $\mathrm{Pt}$ nanoparticles on carbon nanotubes for glucose detection. Electroanalysis. 2007, 19, 717-722.

90. Shirsat, M.D.; Too, C.O.; Wallace, G.G. Amperometric glucose biosensor on layer by layer assembled carbon nanotube and polypyrrole multilayer film. Electroanalysis. 2008, 20, 150-156.

91. Qu, F.; Yang, M.; Chen, J.; Shen, G.; Yu, R. Amperometric biosensors for glucose based on layerby-layer assembled functionalized carbon nanotube and poly (neutral red) multilayer film. Anal. Lett. 2006, 39, 1785-1799.

92. Yao, Y.L.; Shiu, K.K. Low potential detection of glucose at carbon nanotube modified glassy carbon electrode with electropolymerized poly(toluidine blue O) film. Anal. Chim. Acta 2007, 53, 278-284.

93. Zhu, L.; Zhai, J.; Guo, Y.; Tian, C.; Yang, R. Amperometric glucose biosensors based on integration of glucose oxidase onto prussian blue/carbon nanotubes nanocomposite electrodes. Electroanalysis 2006, 18, 1842-1846.

94. Zoua, Y.; Xiang, C.; Suna, L.; Xu, F. Amperometric glucose biosensor prepared with biocompatible material and carbon nanotube by layer-by-layer self-assembly technique. Anal. Chim. Acta 2008, 53, 4089-4095.

95. Zeng, J.; Wei, W.; Liu, X.; Wang, Y.; Luo, G. A simple method to fabricate a Prussian Blue nanoparticles/carbon nanotubes/poly (1,2-diaminobenzene) based glucose biosensor. Microchim. Acta 2008, 160, 261-267.

96. Manesh, K.M.; Kim, H.T.; Santhosh, P.; Gopalan, A.I.; Lee, K. A novel glucose biosensor based on immobilization of glucose oxidase into multiwall carbon nanotubes-polyelectrolyte-loaded electrospun nanofibrous membrane. Biosens. Bioelectron. 2008, 23, 771-779.

97. Choi, H.N.; Han, J.H.; Park, J.A.; Lee, J.M.; Lee, W.Y. Amperometric glucose biosensor based on glucose oxidase encapsulated in carbon nanotube-titania-Nafion composite film on platinized glassy carbon electrode. Electroanalysis 2007, 19, 1757-1763.

98. Manso, J.; Mena, M.L.; Sedeño, P.Y.; Pingarro'n, J. Electrochemical biosensors based on colloidal gold-carbon nanotubes composite electrodes. J. Electroanal. Chem. 2007, 603, 1-7.

99. Manso, J.; Mena, M.L.; Sedeño, P.Y.; Pingarr'on, J.M. Alcohol dehydrogenase amperometric biosensor based on a colloidal gold-carbon nanotubes composite electrode. Electrochim. Acta 2008, 53, 4007-4012.

100. Chu, X.; Duan, D.; Shen, G.; Yu, R. Amperometric glucose biosensor based on electrodeposition of platinum nanoparticles onto covalently immobilized carbon nanotube electrode. Talanta 2007, 71, 2040-2047. 
101. Zou, Y.; Xiang, C.; Suna, L.X.; Xu, F. Glucose biosensor based on electrodeposition of platinum nanoparticles onto carbon nanotubes and immobilizing enzyme with chitosan-SiO2 sol-gel. Biosens. Bioelectron. 2008, 23, 1010-1016.

102. Zhao, K.; Zhuang, S.; Chang, Z.; Songm, H.; Dai, L.; He, P.; Fang, Y. Amperometric glucose biosensor based on platinum nanoparticles combined aligned carbon nanotubes electrode. Electroanalysis 2007, 19, 1069-1074.

103. Kang, X.; Mai, Z.; Zou, X.; Cai, P.; Mo, J. A novel glucose biosensor based on immobilization of glucose oxidase in chitosan on a glassy carbon electrode modified with gold-platinum alloy nanoparticles/multiwall carbon nanotubes. Anal. Biochem. 2007, 369, 71-79.

104. Yang, M.; Yang, Y.; Liu, Y.; Shen, G. Yu, R. Platinum nanoparticles-doped sol-gel/carbon nanotubes composite electrochemical sensors and biosensors. Biosens. Bioelectron. 2006, 21, 1125-1131.

105. Luque, G.L.; Ferreyra, N.F.; Rivas, G.A. Glucose biosensor based on the use of a carbon nanotube paste electrode modified with metallic particles. Microchim. Acta 2006, 152, 277-283.

106. Yao, Y.; Shiu, K.K. Electron-transfer properties of different carbon nanotube materials, and their use in glucose biosensors. Anal. Bioanal. Chem. 2007, 387, 303-309.

107. Jia, J.; Guan, W.; Sim, M.; Li, Y.; Li, H. Carbon nanotubes based glucose needle-type Biosensor. Sensors 2008, 8, 1712-1718.

108. Zhu, L.; Yang, R.; Zhai, J.; Tian, C. Bienzymatic glucose biosensor based on co-immobilization of peroxidase and glucose oxidase on a carbon nanotubes electrode. Biosens. Bioelectron. 2007, 23, 528-535.

109. Chen, L.; Lu, G. Novel amperometric biosensor based on composite film assembled by polyelectrolyte-surfactant polymer, carbon nanotubes and hemoglobin. Biosens. Bioelectron. 2007, 121, 423-429.

110. Chen, S.; Yuan, R.; Chai, Y.; Zhang, L.; Wang, N.; Li, X. Amperometric third-generation hydrogen peroxide biosensor based on the immobilization of hemoglobin on multiwall carbon nanotubes and gold colloidal nanoparticles. Biosens. Bioelectron. 2007, 22, 1268-1274.

111. Tripathi, V.S.; Kandimalla,V.B.; Ju, H. Amperometric biosensor for hydrogen peroxide based on ferrocene-bovine serum albumin and multiwall carbon nanotube modified ormosil composite. Biosens. Bioelectron. 2006, 21, 1529-1535.

112. Luo, X.; Killard, A.J.; Morrin, A.; Smyth, M.R. Enhancement of a conducting polymer-based biosensor using carbon nanotube-doped polyaniline. Anal. Chim. Acta 2006, 575, 39-44.

113. Liu, Y.; Lei, J.; Ju, H. Amperometric sensor for hydrogen peroxide based on electric wire composed of horseradish peroxidase and toluidine blue-multiwalled carbon nanotubes nanocomposite. Talanta 2008, 74, 965-970.

114. Qian, L.; Yang, X. Composite film of carbon nanotubes and chitosan for preparation of amperometric hydrogen peroxide biosensor. Talanta 2006, 68, 721-727.

115. Sa'nchez, S.; Pumera, M.; Cabruja, E.; Fa'bregas, E. Carbon nanotube/polysulfone composite screen-printed electrochemical enzyme biosensors. Analyst 2007, 132, 142-147.

116. Qu, S.; Wang, J.; Kong, J.; Yang, P.; Chen, G. Magnetic loading of carbon nanotube/nano-Fe3O4 composite for electrochemical sensing. Talanta 2007, 71, 1096-1102. 
117. Choi, H. N.; Lyu, Y.K.; Han, J. H.; Lee, W.Y. Amperometric ethanol biosensor based on carbon nanotubes dispersed in sol - gel-derived titania - nafion composite film. Electroanalysis 2007, 19, $1524-1530$.

118. Santos, A.S.; Pereira, A.C.; Dur'an, N.; Kubota, L.T. Amperometric biosensor for ethanol based on co-immobilization of alcohol dehydrogenase and Meldola's Blue on multi-wall carbon nanotube. Electrochim. Acta 2006, 52, 215-220.

119. Du, P.; Liu, S.; Wu, P.; Cai, C. Single-walled carbon nanotubes functionalized with poly(nile blue A) and their application to dehydrogenase-based biosensors. Electrochim. Acta 2007, 53, 1811-1823.

120. Liu, S.; Cai, C. Immobilization and characterization of alcohol dehydrogenase on single-walled carbon nanotubes and its application in sensing ethanol. J. Electroanal. Chem. 2007, 602, 103114.

121. Yang, M.; Yang, Y.; Yang, H.; Shen, G.; Yu, R. Layer-by-layer self-assembled multilayer films of carbon nanotubes and platinum nanoparticles withpolyelectrolyte for the fabrication of biosensors. Biomaterials 2006, 27, 246-255.

122. Tang, L.; Zhu, Y.; Xu, L.; Yang, X.; Li, C. Amperometric glutamate biosensor based on selfassembling glutamate dehydrogenase and dendrimer-encapsulated platinum nanoparticles onto carbon nanotubes. Talanta 2007, 73, 438-443.

123. Wang, J.; Liu, G.; Lin, Y. Amperometric choline biosensor fabricated through electrostatic assembly of bienzyme/polyelectrolyte hybrid layers on carbon nanotubes. Analyst, 2006, 131, 477-483.

124. Song, Z.; Huang, J.D.; Wu, B.Y.; Shi, H.B.; Anzai, J.I.; Chen, Q. Amperometric aqueous sol-gel biosensor for low-potential stable choline detection at multi-wall carbon nanotube modified platinum electrode. Sens. Actuat. B-Chem. 2006, 115, 626-633.

125. Du, D.; Huang, X.; Cai, J.; Zhang, A.; Ding, J.; Chen, S. An amperometric acetylthiocholine sensor based on immobilization of acetylcholinesterase on a multiwall carbon nanotube-crosslinked chitosan composite. Anal. Bioanal. Chem. 2007, 387, 1059-1065.

126. Lee, Y.J.; Lyu, Y.K.; Choi, H.N.; Lee, W.Y. Amperometric tyrosinase biosensor based on carbon nanotube - titania - nafion composite film. Electroanalysis 2007, 19, 1048-1054.

127. Santos, A.S.; Pereira, A.C.; Sotomayor, M.D.P.T.; Tarley, C.R.T.; Dura'n, N.; Kubota, L.T. Determination of phenolic compounds based on co-immobilization of methylene blue and HRP on multi-wall carbon nanotubes. Electroanalysis 2007, 19, 549-554.

128. López, P.B.; Sola, J.; Alegret, S.; MerkoÅi, A. A carbon nanotube PVC based matrix modified with glutaraldehyde suitable for biosensor applications. Electroanalysis 2008, 20, 603-610.

129. Male, K.B.; Hrapovic, S.; Santini, J.M.; Luong, J.H.T. Biosensor for arsenite using arsenite oxidase and multiwalled carbon nanotube modified electrodes. Anal. Chem. 2007, 79, 78317837.

130. Mita, D.G.; Attanasio, A.; Arduini, F.; Diano, N.; Grano, V.; Bencivenga, U.; Rossi, S.; Aminee, A.; Moscone, D. Enzymatic determination of BPA by means of tyrosinase immobilized on different carbon carriers. Biosens. Bioelectron. 2007, 23, 60-65. 
131. Liu, G.; Lin, Y. Biosensor based on self-assembling acetylcholinesterase on carbon nanotubes for flow injection/amperometric detection of organophosphate pesticides and nerve Agents. Anal. Chem. 2006, 78, 835-843.

132. Rahman, M.M.; Shiddiky, M.J.A.; Rahman, M.A.; Shim, Y.B. A lactate biosensor based on lactate dehydrogenase/nictotinamide adenine dinucleotide (oxidized form) immobilized on a conducting polymer/multiwall carbon nanotube composite film. Anal. Biochem. 2009, 384, 159165

133. Williams, K.A.; Veenhuizen, P.T.M.; Torre, B.G.; Eritja, R.; Dekker, C. Carbon nanotubes with DNA recognition. Nature 2002, 420, 761.

134. Baker, S.E.; Cai, W.; Lasseter, T.L.; Weidkamp, K.P.; Hamers, R.J. Covalently bonded adducts of deoxyribonucleic acid (DNA) oligonucleotides with single-wall carbon nanotubes: synthesis and hybridization. Nano Lett. 2002, 2, 1413-1417.

135. Yang, Y.; Wang, Z.; Yang, M.; Li, J.; Zheng, F.; Shen, G.; Yu, R. Electrical detection of deoxyribonucleic acid hybridization based on carbon-nanotubes/nano zirconium dioxide/chitosan-modified electrodes. Anal. Chim. Acta 2007, 584, 268-274.

136. Zhang, W.; Yang, T.; Huang, D. M.; Jiao, K. Electrochemical sensing of DNA immobilization and hybridization based on carbon nanotubes/nano zinc oxide/chitosan composite film. Chin. Chem. Lett. 2008, 19, 589-591.

137. Ma, H.; Zhang, L.; Pan, Y.; Zhang, K.; Zhang, Y. A Novel electrochemical DNA biosensor fabricated with layer-by-layer covalent attachment of multiwalled carbon nanotubes and gold nanoparticles. Electroanalysis 2008, 20, 1220-1226.

138. Niu, S.; Zhao, M.; Hu, L.; Zhang, S.; Carbon nanotube-enhanced DNA biosensor for DNA hybridization detection using rutin-Mn as electrochemical indicator. Sens. Actuat. B-Chem. 2008, 135, 200-205.

139. Erdem, A.; Papakonstantinou, P.; Murphy, H. Direct DNA hybridization at disposable graphite electrodes modified with carbon nanotubes. Anal. Chem. 2006, 78, 6656-6659.

140. Xu, Y.; Ye, X.; Yang, L.; He, P.; Fang, Y. Impedance DNA Biosensor using electropolymerized polypyrrole/multiwalled carbon nanotubes modified electrode. Electroanalysis 2006, 18, 14711478.

141. Qi, H.; Li, X.; Chen, Pei Zhang, C. Electrochemical detection of DNA hybridization based on polypyrrole/ss-DNA/multi-wall carbon nanotubes paste electrode. Talanta 2007, 72, 1030-1035.

142. Chang, Z.; Fan, H.; Zhao, K.; Chen, M.; He, P.; Fang, Y. Electrochemical DNA biosensors based on palladium nanoparticles combined with carbon nanotubes. Electroanalysis 2008, 20, 131-136.

143. Yun, Y.H.; Bange, A.; Heineman, W.R.; Halsall, H.B.; Shanov, V.N.; Dong, Z.; Pixley, S.; Behbehani, M.; Jazieh, A.; Tu, Y.; Wong, D.K.Y.; Bhattacharya, A.; Schulz, M.J. A nanotube array immunosensor for direct electrochemical detection of antigen-antibody binding. Sens. Actuat. B-Chem. 2007, 123, 177-182.

144. Okuno, J.; Maehashi, K.; Kerman, K.; Takamura, Y.; Matsumoto, K.; Tamiya, E. Label-free immunosensor for prostate-specific antigen based on single-walled carbon nanotube arraymodified microelectrodes. Biosens. Bioelectron. 2007, 22, 2377-2381.

145. Yu, X.; Munge, B.; Patel, V.; Jensen, G.; Bhirde, A.; Gong, J.D.; Kim, S.N.; Gillespie, J.; Gutkind, J.S.; Papadimitrakopoulos, F.; Rusling, J.F. Carbon nanotube amplification strategies 
for highly sensitive immunodetection of cancer Biomarkers. J. Am. Chem. Soc. 2006, 128, 11199-11205.

146. Cataldo, V.; Vaze, A.; Rusling, J.F. Improved detection limit and stability of amperometric carbon nanotube-based immunosensors by crosslinking antibodies with polylysine. Electroanalysis 2008, 20, 115-122.

147. Viswanathan, S.; Wu, L.; Huang, M.R.; Ho, J.A. Electrochemical immunosensor for cholera toxin using liposomes and poly (3,4-ethylenedioxythiophene)-coated carbon nanotubes. Anal. Chem. 2006, 78, 1115-1121.

148. S'anchez, S.; Pumera, M.; F’abregas, E. Carbon nanotube/polysulfone screen-printed electrochemical immunosensor. Biosens. Bioelectron. 2007, 23, 332-340.

149. Li, N.; Yuan, R.; Chai, Y.; Chen, S.; An, H. Sensitive immunoassay of human chorionic gonadotrophin based on multi-walled carbon nanotube-chitosan matrix. Bioprocess Biosyst. Eng. 2008, 31, 551-558.

150. Ou, C.; Yuan, R.; Chai, Y.; Tang, M.; Chai, R.; He, X. A novel amperometric immunosensor based on layer-by-layer assembly of gold nanoparticles-multi-walled carbon nanotubes-thionine multilayer films on polyelectrolyte surface. Anal. Chim. Acta 2007, 603, 205-213.

151. Aziz, M.A.; Park, S.; Jon, S.; Yang, H. Amperometric immunosensing using an indium tin oxide electrode modified with multi-walled carbon nanotube and poly (ethylene glycol)-silane copolymer. Chem. Commun. 2007, 25, 2610-2612.

152. Aziz, M.A.; Yang, H. Electrochemical immunosensor using the modification of an aminefunctionalized indium tin oxide electrode with carboxylated single-walled carbon nanotubes. Bull. Kor. Chem.Soc. 2007, 28, 1171 -1174.

(C) 2009 by the authors; licensee Molecular Diversity Preservation International, Basel, Switzerland. This article is an open-access article distributed under the terms and conditions of the Creative Commons Attribution license (http://creativecommons.org/licenses/by/3.0/). 